SAND $-90-8015$

SAND90-8015

DE91 004146

Unlimited Release

Printed November 1990

\title{
DYNAMIC STABILITY OF A SPINNING TUBE CONVEYING A FLUID THROUGH A SYMMETRICAL NONCIRCULAR CROSS-SECTION
}

\author{
G. A. Benedetti \\ Solid Mechanics Division \\ Sandia National Laboratories \\ Livermore, California
}

\begin{abstract}
When a fluid flows inside a tube, the deformations of the tube can interact with the fluid flowing within it and these dynarnic interactions can result in significant lateral motions of the tube and the flowing fluid.

The purpose of this report is to examine the dynamic stability of a spinning tube through which an incompressible frictionless fluid is flowing. The tube can be considered as either a hollow beam or a hollow ble. The analytical results can be applied to spinning or stationary tubes through whici. fluids are transferred; e.g., liquid coolants, fuels and lubricants, slurry solutions, and high explosives in paste form.

The coupled partial differential equations are determined for the lateral motion of a spinning Bernoulli-Euler beam or a spinning cable carrying an incompressible flowing fluid. The beam, which spins about an axis parallel to its longitudinal axis and which can also be loaded by a constant axial force, is straight, uniform, simply supported, and rests on a massless, uniform elastic foundation that spins with the beam. Damping for the beam and foundation is considered by using a combined uniform viscous damping coefficient. The fluid, in addition to being incompressible, is frictionless, has a constant density, and flows at a constant speed relative to the longitudinal beam axis.
\end{abstract}

The Galerkin method is used to reduce the coupled partial differential equations for the lateral motion of the spinning beam to a coupled set of $2 N$, second order, ordinary differential equations for the generalized beam coordinates. By simplifying these equations and examining the roots of the characteristic equation, an analytical solution is obtained for 
the lateral dynamic instability of the beam (or cable). The analytical solutions determine the minimum critical fluid speed and the critical spin speeds, for a specified fluid speed, in terms of the physical parameters of the system.

When the beam (or cable) is not spinning, a certain fluid speed results in a dynamic instability that is analogous to static buckling (divergence), and consequently there are no oscillatory lateral motions associated with the dynamic instability. In this case, when the fluid speed equals (or exceeds) the minimum critical fluid speed, the iateral stiffness of the beam is zero (or negative) and dynamic buckling occurs. For this case the critical speed for the fluid is independent of the viscous damping factor for the beam and foundation.

On the other hand, when the beam is spinning with a certain constant angular speed and the viscous damping factors for the beam and foundation are zero, the dynamic instability is identical to resonance. In this case, as the fluid speed increases, the natural frequencies associated with lateral motion of the beam decrease and small spin speeds of the beam can result in violent self-excited vibrations. When the fluid speed equals or exceeds the minimum critical fluid speed, resonance occurs for any spin speed greater than zero.

For each mode of lateral vibration, resonance can occur at two critical spin speeds provided the fluid speed is less than or equal to the minimum critical fluid speed. The two apparent resonant frequencies for the $N^{\text {th }}$ mode of lateral vibration consist of a certain combination of the two critical spin speeds associated with resonance for the $N^{\text {th }}$ mode of vibration. The critical spin speecis are determined in terms of the natural lateral frequencies for a nonspinning beam (or cable), the fluid speed, and the ratio of fluid mass to beam mass. 


\section{CONTENTS}

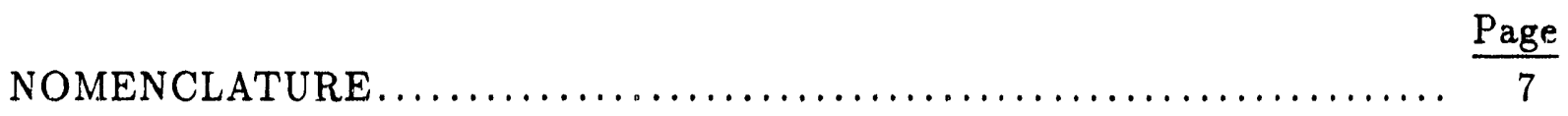

INTRODUCTION $\ldots \ldots \ldots \ldots \ldots \ldots \ldots \ldots \ldots \ldots \ldots \ldots \ldots \ldots \ldots \ldots \ldots \ldots \ldots \ldots \ldots \ldots$

EQUATIONS OF MOTION $\ldots \ldots \ldots \ldots \ldots \ldots \ldots \ldots \ldots \ldots \ldots \ldots \ldots \ldots, 12$

Idealized System $\ldots \ldots \ldots \ldots \ldots \ldots \ldots \ldots \ldots \ldots \ldots \ldots \ldots \ldots \ldots \ldots,{ } 12$

Partial Differential Equations of Motion for the Beam ................. 13

Acceleration of the Fluid and the Loading Function for the Beam ........ 14

Reduction of the Partial Differential Equations of Motion for the

Beam-Fluid System to $2 N$, Second Order, Coupled, Ordinary

Differential Equations..................................... 16

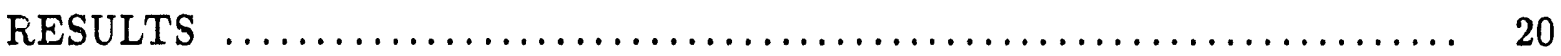

Divergence for a Nonrotating Beam $\ldots \ldots \ldots \ldots \ldots \ldots \ldots \ldots \ldots \ldots \ldots, 20$

Static Buckling ..................................... 22

Divergence for a Nonrotating Cable ............................ 23

Resonance for a Rotating Beam or a Rotating Cable with

Unequal Lateral Stiffness in the $y$ and $z$ Directions .............. 25

Case 1: Fluid Mass is Zero .............................. 29

Case 2: Fluid Speed is Zero ............................. 29

Case 3: Fluid Speed is Less than the Critical Fluid Speed ........... 29

Case 4: Fluid Speed Equals the Critical Fluid Speed ............... 29

Case 5: Fluid Speed Exceeds the Critical Fluid Speed ............. 30

Frequency of Lateral Vibration at Resonance .................. 31

Case A: Spin Speed Equal to First Critical Speed for $N^{\text {th }}$ Mode .. 31

Case B: Spin Speed Equal to Second Critical Speed for $N^{\text {th }}$ Mode 32

Comparison Between Experimental Measurements and Predicted Values .. 33

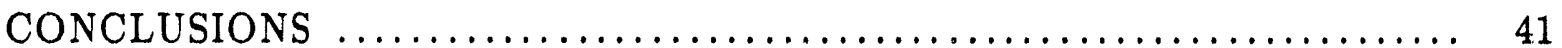

Nonrotating Beam or Cable .............................. 41

Rotating Beam or Cable $\ldots \ldots \ldots \ldots \ldots \ldots \ldots \ldots \ldots \ldots \ldots \ldots \ldots \ldots, 42$

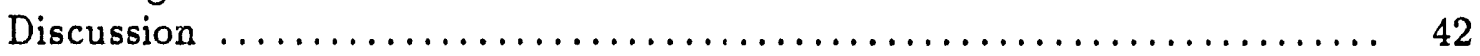

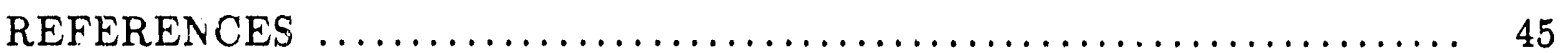




\section{NOMENCLA'TURE}

$a$

$\bar{a}_{f}$

A

$C_{n}$

$E$

$f_{n}, q_{n}$

$\bar{i}, \bar{j}, k$

I

$I_{y}, I_{z}$

$k$

$k_{y}, k_{z}$

$\ell$

$m$

$m_{f}$

$N$

l)

$P_{c r}$

$P_{e}=E I(\pi / \ell)^{2}$

$P^{*}=P / P_{e}$

$q_{p}$ offset distance

acceleration vector for fluid

cross-sectional area of beam

$n^{\text {th }}$ combined viscous damping coefficient for beam and foundation per unit length

modulus of elasticity

generalized beam coordinates for the $y$ and $z$ directions, respectively

unit vectors along the body fixed $x, y, z$ axes, respectively

area moment of inertia of beam cross-section (symmetrical) about $y$ or $z$ axes originating at centroid of beam cross-section

area moment of inertia of beam cross-section about $y$ and $z$ i xes, respectively, originating at centroid of beam cross-section

foundation stiffness per unit length

foundation stiffness per unit length in the $y$ and $z$ directions, respectively

beam length

mass of beam or cable per unit length

mass of fluid per unit length

number of generalized beam coordinates

constant axial force applied at the end of a beam in the $x$ direction through the centroid of the cross-section

minimum static buckling load (defined as static buckling load)

magnitude of the Euler buckling load

nondimensional axial force

generalized beam coordinate 


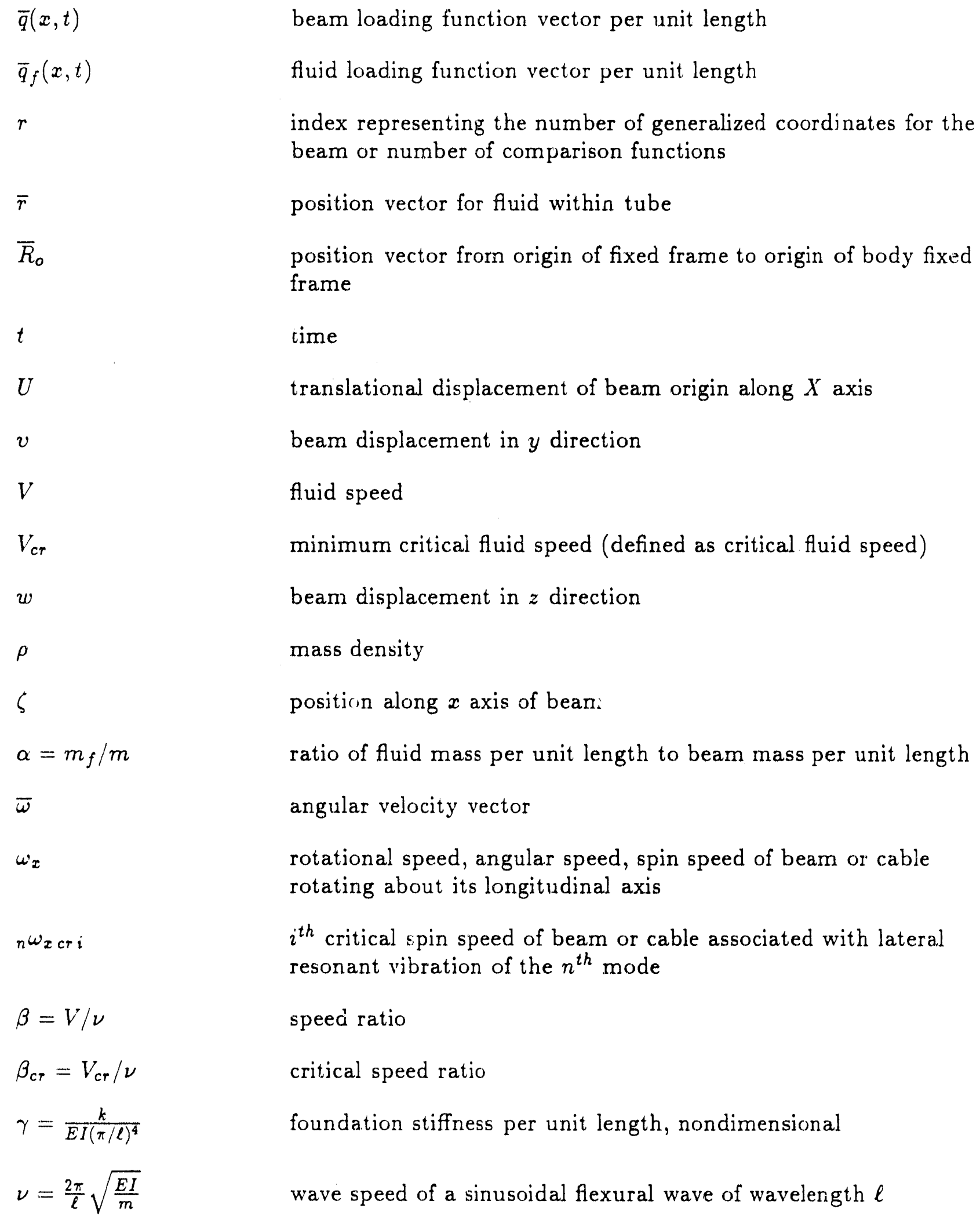


$\xi_{n}=\frac{C_{n}}{2 m \omega_{n}}$

$\omega_{n}=$

$\sqrt{\frac{E I}{m}\left(\frac{\pi}{\ell}\right)^{4}\left[n^{4}+n^{2} \frac{P}{P_{e}}+\gamma\right]}$ $n^{\text {th }}$ undamped natural circular frequency of lateral vibration for a uniform, simply supported beam on an elastic foundation with a positive (tensile) axial force applied at its end

${ }_{1} \Omega_{N}^{\text {apparent }},{ }_{2} \Omega_{N}^{\text {apparent }}$

$\Omega_{N y}, \Omega_{N z}$

$n^{\text {th }}$ damping factor for beam and foundation or ratio of the $n^{\text {th }}$ viscous damping coefficient to the critical viscous damping coefficient for the $n^{\text {th }}$ mode

first and second circular frequencies associated with the $N^{\text {th }}$ mode of lateral vibration for the beam (or cable)

natural bending cicular frequencies associated with the $N^{\text {th }}$ mode of lateral vibration for the beam (or cable) in the $y$ and $z$ directions, respectively, when the fluid is flowing along the beam with a constant speed $V\left(V<V_{c r}\right)$ and the beam is not spinning (i.e., $\omega_{x}=0$ ); e.g.,

$$
\Omega_{N y}=\sqrt{\frac{\omega_{N y}^{2} \cdot \alpha(N \pi / \ell)^{2} V^{2}}{1+\alpha}}
$$

differentiation with respect to time, $t$ 


\section{INTRODUCTION}

When a fluid flows over the external surfaces of a structure, dynamic interactions between the fluid and structure can occur that can result in significant motions of the structure. Some examples are the vibration of bridges, tall chimneys and towers, electrical transmission lines and the "flutter" of aircraft wings.

When a fluirl flows inside a tube, the deformations of the tube can interact with the fluid flowing within it and these dynamic interactions can result in significant lateral motions of the tube and flowing fluid.

The purpose of this report is to examine the dynamic stability of a spinning tube, with a symmetrical noncircular cross-section, through which an incompressible frictionless fluid is flowing. The tube can be considered as either a hollow beam or a hollow cable. The analytical results can be applied to spinning or stationary tubes through which fluids are transferred; e.g., liquid coolants, fue's and lubricants, slurry solutions, high explosives in paste form, etc. Fluids transferred from one location to another along the spin axis of a spinning body such as an artillery projectile is another example.

An analytical solution is obtained that determines the critical fluid speed as a function of the physical parameters for the system. Also, the critical angular speeds for resonance as well as the frequencies associ ted with resonant lateral vibration are determined in terms of the system's physical parameters and a specified fluid speed.

When the tube is not spinning, a certain fluid speed results in dynamic instability, which is analogous to static buckling (divergence) and consequertly, there are no oscillatory lateral motions associated with the dynamic instability. In this case, when the fluid speed equals (or exceeds) the minimum critical fluid speed, the lateral stiffness of the tube is zero (or negative) and dynamic buckling occurs. For this case, the critical speed for the fluid is independent of the damping factor for the beam and foundation.

On the other hand, when the tube is spinning with a certain constant angular speed and the viscous damping factors for the beam and foundation are zero, the dynamic instability is identical to resonance. In this case, as the fluid speed increases, the natural frequencies associated with lateral motion of the tube decrease and small spin speeds or angular speeds of the tube can resuli in violent self-excited vibrations. When the fluid speed equals or exceeds the minimum critical speed, resonance occurs for any spin speed greater than zero. If damping is present, large amplitude lateral vibrations can occur at or near the critical spin speeds.

For each mode of lateral vibration, resonance can occur at two critical spin speeds provided the fluid speet is less than or equal to the minimum critical fluid speed. The apparent resonant frequencies for the $N^{\text {th }}$ mode of lateral vibration consist of a certain combination of the two critical spin speeds associated with resonance for the $N^{\text {th }}$ mode of vibration.

The critical spin speeds are determined in terms of the natural lateral frequencies for a nonspinning beam (or cable), the fluid speed, and the ratio of fluid mass to beam mass. 


\section{EQUATIONS OF MOTION}

\section{Idealized System}

The idealized system is shown in Figure 1 and consists of a fluid flowing through an elastic tube of constant cross-section. Consider the case where the tube cross-section is symmetrical with respect to the rotating body fixed $y$ and $z$ axes but the lateral stiffnesses in the $y$ and $z$ directions are different; e.g., a beam with an elliptical or rectangular cross-section.

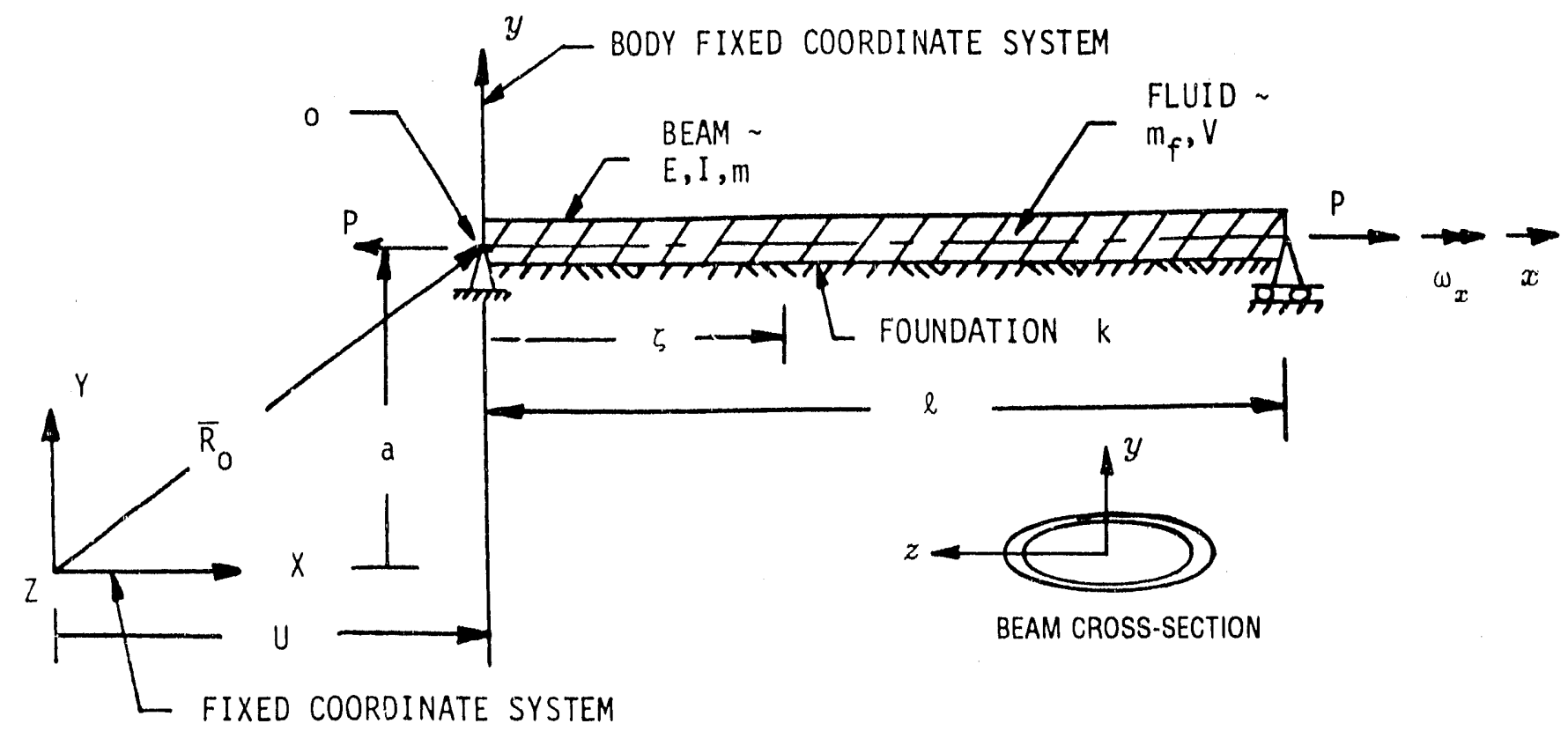

Figure 1. Idealized syste..1.

The tube is modeled as a straight, simply supported, Bernoulli-Euler beam of finite length supported on a continuous, linear-elastic foundation. The beam is continuous, homogeneous, and isotropic with a linear constitutive relationship between stress and strain. The foundation modulus is constant and the force per unit length exerted on the beam by the foundation is directly proportional to the transverse beam deflection. The mass of the foundation is neglected. Constant viscous damping coefficients are assumed for the beam and foundation and the darnping forces are linear with respect to the transverse beam velocity. The beam and foundation are rotating with a constant angular speed $\omega_{x}$ as well as translating with a constant speed, $\dot{U}$. In addition, the beam is 
subjected to a constant axial force $P$ applied at the end of the beam through the centroid of the cross-section in the $x$ direction.

The fluid is flowing in the $x$ direction at a constant speed $V$ relative to the longitudinal beam axis. In addition, the fluid is incompressible and has a constant density. Friction as well as gravity is neglected. The fluid is supplied from a reservoir and is always flowing at a constant speed during the time of interest.

\section{Partial Differential Equations of Motion For The Beam}

The partial differential equations which describe the lateral motions of the beam, in the body fixed $y$ and $z$ directions, are derived in Reference 1 and are expressed as 1

$$
\begin{aligned}
E I_{z} v^{\prime \prime \prime \prime} & -\left[P+\rho I_{z} \omega_{x}^{2}\right] v^{\prime \prime}+\left[k_{y}-m \omega_{x}^{2}\right] v+m \ddot{v}-\rho I_{z} \ddot{v}^{\prime \prime} \\
& +C_{n} \dot{v}-2 m \omega_{x}^{2} \dot{w}=m a \omega_{x}^{2}+q_{y}(x, t) \\
E I_{y} w^{\prime \prime \prime \prime}-\left[P+\rho I_{y} \omega_{x}^{2}\right] w^{\prime \prime}+\left[k_{z}-m \omega_{x}^{2}\right] w+m \ddot{w}-\rho I_{y} \ddot{w}^{\prime \prime} & -C_{n} \dot{w}+2 m \omega_{x} \dot{v}=q_{z}(x, t)
\end{aligned}
$$

The lateral displacements of the bearn, $v$ and $\omega$, in the $y$ and $z$ directions, respectively, are functions of the space coordinate $x$ and time $t$, and

$$
()^{\prime}=\frac{\partial()}{\partial x}, \quad(\dot{)})=\frac{\partial()}{\partial t} .
$$

Also, $q_{y}(x, t)$ and $g_{z}(x, t)$ represent the beam loading functions per unit length in the positive $y$ and $z$ directions, respectively. Noie that Eqs. (1) and (2) are coupled due to the Coriolis effect.

The boundary and initial conditions associated with Eqs. (1) and (2) are expressed by the following set of equations:

At $x=0$ :

$$
\begin{aligned}
v(0, t) & =0 \\
v^{\prime \prime}(0, t) & =0 \\
w(0, t) & =0 \\
w^{\prime \prime}(0, t) & =0
\end{aligned}
$$

1 Note that when $I_{y}$ and $I_{z}$ equal zero, Eqs. (1) and (2) reduce to those for a uniform fixed-fixed cable. That is, the beam bending stiffness is zero. 
At $x=\ell$ :

$$
\begin{aligned}
v(\ell, t) & =0 \\
v^{\prime \prime}(\ell, t) & =0 \\
w(\ell, t) & =0 \\
w^{\prime \prime}(\ell, t) & =0
\end{aligned}
$$

At $t=0$ :

$$
\begin{gathered}
v(x, 0)=g_{1}(x) \\
\dot{v}(x, 0)=g_{2}(x) \\
w(x, 0)=h_{1}(x) \\
\dot{w}(x, 0)=h_{2}(x)
\end{gathered}
$$

\section{Acceleration of the Fluid and the Loading Function for the Beam}

Significant dynamic interactions can occur between the flowing fluid and the beam deformations. To determine these interactions $i+$ is necessary to examine the kinematics of the fluid while the fluid is in contact with the beam.

The absolute acceleration of the center of mass of a fluid element located at any position $\zeta$ along the beam axis is given by

$$
\bar{a}_{f}=\ddot{\bar{R}}_{o}+\dot{\bar{\omega}} \times \bar{r}+2 \bar{\omega} \times \dot{\bar{r}}_{r e l}+\ddot{\bar{r}}_{r e l}+\bar{\omega} \times(\bar{\omega} \times \bar{r})
$$

The acceleration of the origin of the body fixed $x y z$ system is given by

$$
\ddot{\bar{R}}_{o}=\dot{\bar{\omega}} \times \bar{R}_{o}+2 \bar{\omega} \times \dot{\bar{R}}_{o_{\text {rel }}}+\ddot{\bar{R}}_{o_{\text {rel }}}+\bar{\omega} \times\left(\bar{\omega} \times \bar{R}_{o}\right)
$$

where, for the particuiar case under consideration,

$$
\begin{aligned}
\bar{R}_{o} & =U \bar{i}+a \bar{j} \\
\dot{\bar{R}}_{o_{r e l}} & =\dot{U} \bar{i} \\
\ddot{\bar{R}}_{0_{r e l}} & =0 \\
\bar{\omega} & =\omega_{z} \bar{i} \\
\dot{\bar{\omega}} & =0 .
\end{aligned}
$$


The position vector from the origin of the $x y z$ system to the center of mass of a fluid element located at any position along the beam axis is given by

$$
\bar{r}=\zeta \bar{i}+v \bar{j}+w \bar{k}
$$

Since the fluid lateral displacements $v$ and $w$ depend on $x$ and $t$, i.e. $v=v(x, t)$ and $w=w(x, t)$, the expressions for $\dot{\bar{r}}_{r e l}$ and $\ddot{\bar{r}}_{r e l}$ for the fluid in contact with the beam are given by

$$
\begin{gathered}
\dot{\bar{r}}_{\text {rel }}=\frac{a \zeta}{d t} \bar{i}+\left[\frac{\partial v}{\partial x} \frac{d \zeta}{d t}+\frac{\partial v}{\partial t}\right] \bar{j}+\left[\frac{\partial w}{\partial x} \frac{d \zeta}{d t}+\frac{\partial w}{\partial t}\right] \bar{k} \\
\ddot{\bar{r}}_{\text {rel }}=\frac{d^{2} \zeta}{d t^{2}} \bar{i}+\left[\frac{\partial^{2} v}{\partial x^{2}}\left(\frac{d \zeta}{d t}\right)^{2}+2 \frac{\partial^{2} v}{\partial x \partial t} \frac{d \zeta}{d t}+\frac{\partial v}{\partial x} \frac{d^{2} \zeta}{d t^{2}}+\frac{\partial^{2} v}{\partial t^{2}}\right] \bar{j} \\
+\left[\frac{\partial^{2} w}{\partial x^{2}}\left(\frac{d \zeta}{d t}\right)^{2}+2 \frac{\partial^{2} w}{\partial x \partial t} \frac{d \zeta}{d t}+\frac{\partial w}{\partial x} \frac{d^{2} \zeta}{d t^{2}}+\frac{\partial^{2} w}{\partial t^{2}}\right] \bar{k}
\end{gathered}
$$

where

$$
\frac{d \zeta}{d t}=V
$$

the constant velocity of the fluid element relative to the beam along the $x$ axis, and

$$
\frac{d^{2} \zeta}{d t^{2}}=0
$$

the acceleration of the fluid relative to the beam along the $x$ axis.

Some explanation of the terms appearing in Eqs. (10) and (11) may be helpful. Consider the components of velocity and acceleration of the fluid element in the $y$ direction in Eqs. (10) and (11). That is, the terms associated with the coefficient of the unit vector $\bar{j}$. The first term in the coefficient in Eq. (10) is equal to the $y$ component of $V$ and results from the slope of the beam; the second term results from the velocity of the beam in the $y$ direction. In equation (11), the first term in the $\bar{j}$ coefficient is due to the centripetal acceleration of the fluid which results from a change in the direction of $V$ and is caused by the curvature of the beam. The second term is due to the Curiolis acceleration which arises because the fluid is moving with a velocity $V$ relative to the beam while the beam element has an angular velocity $\frac{\partial^{2}}{\partial} \frac{v}{\partial t}$. The third term is equal to the $y$ component of $\dot{V}$ and is zero in this particular case since the acceleration of the fluid relative to the beam aiong the $x$ axis is zero. The fourth term results from the acceleration of the beam in the $y$ direction. Similar statements can be made for the components of velocity and acceleration of the fluid in the $z$ direction.

The force per unit length acting on the fluid is giv an by

$$
\bar{q}_{f}(x, t)=m_{f} \bar{a}_{f}
$$

and the force per unit length act $\because \mathrm{g}$ on the beam is given by 


$$
\bar{q}_{b}(x, t)=-\bar{q}_{f}(x, t)
$$

where

$$
\bar{q}_{b}(x, t)=q_{y}(x, t) \bar{j}+q_{z}(x, t) \bar{k}
$$

provided the fluid is in contact with the beam.

Substitution of Eqs. (7) through (11) into Eq. (6) and utilizing Eqs. (12) through (14) results in the loading function per unit length for the beam, $\bar{q}_{b}(x, t)$. r'he components of the loading function for the beam in the $y$ and $z$ directions are expressed by Eqs. (15) and (16), respectively, and will subsequently be used in Eqs. (1) and (2). Recall that $\zeta$ locates the center of mass of a fluid element along the $x$ axis of the beam between $0 \leq x \leq l$.

$$
\begin{aligned}
q_{y}(x, t)= & m_{f} \omega_{x}^{2} a+m_{f} \omega_{x}^{2} v+2 m_{f} \omega_{x} V w^{\prime}+2 m_{f} \omega_{x} \dot{w} \\
& -m_{f} V^{2} v^{\prime \prime}-2 m_{f} V \dot{v}^{\prime}-m_{f} \ddot{v} \\
q_{z}(x, t)= & m_{f} \omega_{x}^{2} w-2 m_{f} \omega_{x} V v^{\prime}-2 m_{f} \omega_{x} \dot{v}-m_{f} V^{2} w^{\prime \prime} \\
& -2 m_{f} V \dot{w}^{\prime}-m_{f} \ddot{w}
\end{aligned}
$$

Also recall that the speed $V$ of the fluid is constant.

Reduction of the Partial Differential Equations of Motion for the Beam-Fluid System to $2 N$, Second Order, Coupled, Ordinary Differential Equations

Substitution of Eqs. (15) and (16) into Eqs. (1) and (2) results in the equations below, which describe the motions of the beam-fluid system in the $y$ and $z$ directions, respectively, for $0 \leq x \leq \ell$ and $t \geq 0$.

$$
\begin{array}{r}
E I_{z} v^{\prime \prime \prime \prime}-\left[P+\rho I_{z} \omega_{x}^{2}-m_{f} V^{2}\right] v^{\prime \prime}+\left[k_{y}-\left(m+m_{f}\right) \omega_{x}^{2}\right] v \\
-\rho I_{z} \ddot{v}^{\prime \prime}+\left(m+m_{f}\right) \ddot{v}+C_{n} \dot{v}-2 \omega_{x}\left(m+m_{f}\right) \dot{w} \\
+2 m_{f} V \dot{v}^{\prime}-2 m_{f} \omega_{x} V w^{\prime}=a \omega_{x}^{2}\left(m+m_{f}\right) \\
E I_{y} w^{\prime \prime \prime \prime}-\left[P+\rho I_{y} \omega_{x}^{2}-m_{f} V^{2}\right] w^{\prime \prime}+\left[k_{z}-\left(m+m_{f}\right) \omega_{x}^{2}\right] w \\
-\rho I_{y} \ddot{w}^{\prime \prime}+\left(m+m_{f}\right) \ddot{w}+C_{n} \dot{w}+2 \omega_{x}\left(m+m_{f}\right) \dot{v} \\
+2 m_{f} V \dot{w}^{\prime}+2 m_{f} \omega_{x} V v^{\prime}=0
\end{array}
$$


The lateral displacements of the beam are approximated by

$$
\begin{aligned}
& v=\sum_{n=1}^{n=N} \sin \frac{n \pi x}{\ell} f_{n}(t) \\
& w=\sum_{p=1}^{p=N} \sin \frac{p \pi x}{\ell} q_{p}(t)
\end{aligned}
$$

Equations (21) ind (22) result from utilizing the Galerkin method to represent the motion of the beam as a $2 \mathrm{~N}$ degree-of-freedom sys:em

$$
\begin{aligned}
& {\left[\left(m+m_{f}\right)+\rho I_{z}\left(\frac{-\pi}{\ell}\right)^{2}\right] \ddot{f}_{r}(t)+C_{r} \dot{f}_{r}(t)} \\
& -\left\{E I_{z}\left(\frac{r \pi}{\ell}\right)^{4}-\left[P-3 I_{z} \omega_{z}^{2}-m_{f} l^{2}\right]\left(\frac{r \pi}{\varepsilon}\right)^{2}+\left[k_{y}-\left(m+m_{f}\right) \omega_{x}^{2}\right]\right\} f_{r}(t) \\
& -2 \dot{\omega}_{x}\left(m+m_{f}\right) \dot{q}_{r}(t)-\frac{4}{\ell} m_{f} V \sum_{n=1}^{n=N}\left(\frac{n \pi}{\ell}\right) \frac{2 r}{(\pi / \ell)\left(r^{2}-n^{2}\right)} \dot{f}_{n}(t) \\
& -\frac{4}{\ell} m_{f \omega_{z}} r \cdot \sum_{p=1}^{p=N}\left(\frac{p \pi}{\ell}\right) \frac{2 r}{(\pi \ell)\left(r^{2}-p^{2}\right)} q_{p}(t)=a \omega_{z}^{2}\left(m+m_{f}\right)[1-\cos r \pi] \\
& r=1,2,3, \ldots, N \\
& {\left[\left(m-m_{f}\right)+\rho I_{y}\left(\frac{r \pi}{\ell}\right)^{2}\right] \ddot{q}_{r}(t)+C_{r} \dot{g}_{r}(t)} \\
& -\left\{E I_{y}\left(\frac{r \pi}{\ell}\right)^{4}-\left[P-\rho I_{y} \omega_{z}^{2}-m_{f} V^{2}\right]\left(\frac{r \pi}{\ell}\right)^{2}-\left[k_{z}-\left(m+m_{f}\right) \omega_{x}^{2}\right]\right\} q_{r}(t) \\
& -2 \omega_{x}\left(m+m_{f}\right) \dot{f}_{r}(t)+\frac{4}{\ell} m_{f} V \sum_{p=1}^{p=N}\left(\frac{p \pi}{\ell}\right) \frac{2 r}{(\pi / \ell)\left(r^{2}-p^{2}\right)} \dot{q}_{p}(t) \\
& -\frac{4}{\ell} m_{f} \dot{u}_{z} l \cdot \sum_{n=1}^{n=N}\left(\frac{n \pi}{\ell}\right) \frac{2 r}{(\pi, \ell)\left(r^{2} \cdots n^{2}\right)} f_{n}(t)=0 \\
& r=1,2,3, \ldots, N
\end{aligned}
$$

where the last two terms on the left hand side of the equal sign in Eqs. (21) and (22) are nonzero only if $(r-n)$ and $(r-p)$ are odd numbers.

If the off-diagonal term. the diagonal are considered, i.e. $n=p=r=.1$ ), the homogeneous form of Eqs. (21) and $(22)$ can be simplified and expressed as 


$$
\begin{gathered}
{\left[1+\alpha+\frac{\rho I_{z}}{m}\left(\frac{N \pi}{\ell}\right)^{2}\right] \ddot{f}_{N}(t)+2 \omega_{N_{y}} \xi_{N_{y}} \dot{f}_{N}(t)} \\
+\left\{\omega_{N_{y}}^{2}-\alpha\left(\frac{N \pi}{\ell}\right)^{2} V^{2}-\omega_{x}^{2}\left[1+\alpha-\frac{\rho I_{z}}{m}\left(\frac{N \pi}{\ell}\right)^{2}\right]\right\} f_{N}(t) \\
-2 \omega_{x}(1+\alpha) \dot{q}_{N}(t)=0 \\
{\left[1+\alpha+\frac{\rho I_{y}}{m}\left(\frac{N \pi}{\ell}\right)^{2}\right] \ddot{q}_{N}(t)+2 \omega_{N_{\iota}} \xi_{N_{z}} \dot{q}_{N}(t)} \\
+\left\{\omega_{N_{z}}^{2}-\alpha\left(\frac{N \pi}{\ell}\right)^{2} V^{2}-\omega_{x}^{2}\left[1+\alpha-\frac{\rho I_{y}}{n}\left(\frac{N \pi}{\ell}\right)^{2}\right]\right\} q_{N}(t) \\
+2 \omega_{x}(1+\alpha) \dot{f}_{N}(t)=0
\end{gathered}
$$

where

$$
\begin{aligned}
& a=\frac{m_{i}}{m} \\
& P_{e y}=E I_{z}\left(\frac{\pi}{l}\right)^{2} \\
& P_{e z}=E I_{y}\left(\frac{\pi}{i}\right)^{2} \\
& \gamma_{y}=\frac{k_{y}}{E I_{z}(\pi /)^{t}} \\
& \gamma_{z}=\frac{k_{z}}{E I_{y}(\pi / l)^{4}} \\
& \omega^{2} N_{y}=\frac{E I_{z}}{m}\left(\frac{\pi}{\ell}\right)^{4}\left[N^{4}+N^{2} \frac{P}{P_{\varepsilon y}}+\gamma_{y}\right] \\
& \dot{u}_{N_{z}}^{2}=\frac{E I_{y}}{m}\left(\frac{\pi}{\ell}\right)^{4}\left[N^{4}+N^{2} \frac{P}{F_{e_{z}}}+\gamma_{z}\right] \\
& \xi_{N_{y}}=\frac{C_{N}}{2 m \omega N_{y}} \\
& \xi_{N_{s}}=\frac{C_{N_{j}}}{2 m_{N_{N_{z}}}}
\end{aligned}
$$

Since the dynamic stability of Eqs. (21) and (22) is of interest, it is only necessary to consider the homogeneous form of these equations.

The initial conditions associated with Eqs. (23) and (24) are given by 


$$
\begin{aligned}
& f_{N}(0)=\frac{2}{\ell} \int_{x=0}^{x=\ell} g_{1}(x) \sin \frac{N \pi x}{\ell} d x \\
& \dot{f}_{N}(0)=\frac{2}{\ell} \int_{x=0}^{x=\ell} g_{2}(x) \sin \frac{N \pi x}{\ell} d x \\
& q_{N}(0)=\frac{2}{\ell} \int_{x=0}^{x=\ell} h_{1}(x) \sin \frac{N \pi x}{\ell} d x \\
& \dot{q}_{N}(0)=\frac{2}{\ell} \int_{x=0}^{x=\ell} h_{2}(x) \sin \frac{N \pi x}{\ell} d x
\end{aligned}
$$

For reasonable values of $\mathrm{N}$, the terms involving the rotary inertia and the change in direction of the angh'ar momentum, for an element of bearn cross-section, are neglecter. That is, $(\rho I / m)(N \pi / \ell)^{2}$ is very small compared to unity for reasonable values of $N$, where $I=I_{y}$ or $I=I_{z}$. Consequently, the terms involving $(\rho I / m)(N \pi / \ell)^{2}$ in Eqs. (23) and (24) are neglected. 


\section{RESULTS}

\section{Divergence for a Nonrotating Beam}

When the angular speed of the beam is zero (i.e., $\omega_{x}=0$ ), the simplified equations (23) and (24) are not coupled by the Coriolis effect. For this case, dynamic instability in the form of a divergence rather than a resonance will occur when the fluid speed $V$ equals or exceeds a critical fluid speed $V_{c r}$. The incipient critical fluid speed (i.e., on the boundary between stability and instability) is determined by requiring that one of the roots to the characteristic equation, for either Eqs. (23) or (24), equal zero. T'.e other root is negative sirice the viscous damping factor is not zero. Since there are no repeated zero roots to the characteristic equation (i.e., $\xi_{N y} \neq 0$ and $\xi_{N z} \neq 0$ ), divergence occurs when one of the roots is real and positive.

It is important to note that the simplified equations (23) and (24) will each yield a critical fluid speed and that these critical fluid speeds will, in general, not be the same since they depend upon $I_{y}, I_{z}, \gamma_{y}, \gamma_{z}$ and $N$. The minimum critical fluid speed is of interest in this analysis and it is assumed that $\omega_{N y}$ is less than $\omega_{N z}$. This is an arbitrary choice and in actual practice both frequencies need to be determined since the natural bending frequencies are influenced by parameters other than the moments of inertia of area for the beam cross-section. Henceforth in this analysis, the minimum critical fluid speed is defined as the critical fluid speed $V_{c r}$ and is determined from Eq. (23).

Therefore, for incipient divergerice the minimum critical fluid speed, $V_{r . r}$ is determined from Eq. (23) and is obtained from

$$
-2 \omega_{N y} \xi_{N y} \pm\left\{\left(2 \omega_{N y} \xi_{N y}\right)^{2}-4(1+\alpha)\left[\omega_{N y}^{2}-\alpha\left(\frac{N \pi}{\ell}\right)^{2} V_{r .1}^{2}\right]\right\}^{1 / 2}=0
$$

or

$$
V_{c r}=\frac{\ell}{N \pi} \omega_{N y} \sqrt{\frac{1}{\alpha}}
$$

where $\alpha$ is not zero and $\omega_{N_{y}}$ is given by

$$
\omega_{N y}=\sqrt{\frac{E I_{x}}{m}\left(\frac{\pi}{\ell}\right)^{4}\left[N^{4}+N^{2} \frac{P}{P_{e y}}+\gamma_{y}\right]} .
$$

Equations (26) and (26a) are not useful unless the value of $N$ is known since the lowest critical speed may occur with $N=1,2,3, \ldots$, depending upon the values of the other parameters in Eqs. (26) and (26a). Since divergence of the beam oscurs with no oscillatory motion, (i.e., the roots to the characteristic equation are real and not complex conjugates) it appears that the beam behaves as if it were under the influence of its static buckling load when the speed of the fluid $V$ equals the critical speed $V_{c r}$. This 
suggests that the beam is buckling into one of its $N$ modes and that the term containing $V^{2}$ in Eq. (23) is analogous to an equivalent static buckling load when $V$ equals $V_{c r}$. This observation allows the value of $N$ to be determined for use in Eq. (26).

In order to determine the value of $N$ which makes the critical fluid speed $V_{c r}$ a minimum, consider the special case when the nondimensional foundation stiffness $\gamma_{y}=0$. Now', there is no resisting foundation and from Eqs. (26) and (26a), $N$ must equal 1. If $\gamma_{y}$ is very small but greater than zero, $N$ still equals 1 in Eqs. (26) and (26a). Thus, for a very flexible foundation, the beam buckles without an intermediate inflection point. By gradually increasing $\gamma_{y}, V_{c r}$ in Eq. (26) will eventually be smaller for $N=2$ than for $N=1$ (refer to Figure 3). At this value of $\gamma_{y}$ the buckled beam will have an inflection point at $\ell / 2$. The limiting value of $\gamma_{y}$ at which the transition from $N=1$ to $N=2$ occurs is found from the condition that at this limiting value of $\gamma_{y} \mathrm{Eq}$. (26) should give the same value of $V_{c r}$ independent of whether $N=1$ or $N=2$. Therefore, a relationship for the number of half-sine waves $N$ in which the beam subdivides at $V_{c r}$ (dynamic buckling) is obtained by equating $V_{c r}$ for the $N^{\text {th }}$ mode to $V_{c r}$ for the $(N+1)$ mode for Eq. (26).

This minimizes the critical speed in Eq. (26) for these inflection points and results in the inequality

$$
N^{2}(N+1)^{2} \geq \gamma_{y}
$$

where the nondimensional foundation stiffness is given by

$$
\gamma_{y}=\frac{k_{y}}{E I_{z}(\pi / \ell)^{4}}
$$

The first value of $N$ which satisfies the inequality $(27)$ is the $N$ which is used in Eq. (26) to determine $V_{c r}$. Table I illustrates the use of the inequality (27).

TABLE I.

USE OF THE INEQUALITY IN EQ. (27).

\begin{tabular}{c|c}
$\begin{array}{c}\text { Value of Nonndimensional } \\
\text { Foundation Stiffness, } \gamma_{y}\end{array}$ & $\begin{array}{c}\text { Value of } N \text { for } \\
\text { use in Eq. (26) }\end{array}$ \\
\hline $0 \leq \gamma_{y} \leq 4$ & 1 \\
$4 \leq \gamma_{y} \leq 36$ & 2 \\
$36 \leq \gamma_{y} \leq 144$ & 3 \\
$144 \leq \gamma_{y} \leq 400$ & 4 \\
\hline
\end{tabular}


Defining $\nu$ as the wave speed of a sinusoidal flexural wave of length $\ell$ and $\beta_{c r}$ as the critical speed ratio, where

$$
\nu=\frac{2 \pi}{\ell} \sqrt{\frac{E I_{z}}{m}}
$$

and

$$
\beta_{c r}=\frac{V_{c r}}{\nu}
$$

Equation (26) is expressed in nondimensional form as

$$
\beta_{c r}=\sqrt{\frac{1}{4 \alpha}\left[N^{2}+\frac{P}{F_{e y}^{\prime}}+\frac{\gamma_{y}}{N^{2}}\right]}
$$

where $\alpha$ is not zero. A positive value of $P$ in Eq. (29) implies a tensile axial load acting on the end of the beam as shown in Figure 1. Recall that the value of $N$ to be used in Eq. (29) is the first value of $N$ which satisfies the inequality (27). That is,

$$
N^{2}(N+1)^{2} \geq \gamma_{y}
$$

Also note that the critical speed ratio $\beta_{c r}$ is independent of the damping factor $\xi_{N y}$. Equation (29) and the inequality (30) are illustrated in Figure 2 for $P_{y}^{*}=\gamma_{y}=0$ and in Figure 3 for $P_{y}^{*}=0$ and $\alpha=2$.

Static Buckling-If divergence occurs when the critical speed ratio $\left(\beta_{c r}\right)$ equals zero (for finite values of $\alpha$ ), then the axial force $P$ acting on the beam must equal the static buckling load $P_{c r}$. Therefore with $\beta_{c r}=0$, Eq. (29) yields the minimum static buckling load for the beam shown in Figure 1:

$$
P_{c r}=-\left(N^{2}+\frac{\gamma_{y}}{N^{2}}\right) P_{e y}
$$

where $P_{e y}=E I_{z}(\pi / \ell)^{2}$ and the inequality (30) applies. The minus sign in Eq. (31) implies that $P_{c r}$ is compressive and acts in a direction opposite to that shown in Figure 1.

The minimum static buckling load $P_{c r}$ given by Eq. (31) represents static buckling of the beam for lateral beam displacements which occur in the $x-y$ plane. Recall that it is assumed that $\omega_{N y}<\omega_{N z}$.

A larger second static buckling load, which can be determined in a manner analogous to Eq. (31), represents static buckling of the beam for lateral displacements which occur in the $x-z$ plane. Howevor, the minimum static buckling load $P_{c r}$ is of interest in this analysis. Recall that in the case for dynamic instability the minimum critical fluid speed $V_{c r}$ is of interest and that $\omega_{N y}<\omega_{N_{z}}$. 


\section{Divergence of a Nonrotating Cable}

Consider a uniform fixed-fixed cable not on an elastic foundation (i.e., $\gamma_{y}=0$ and $\left.\gamma_{z}=0\right)$ through which an incompressible frictionless fluid is flowing at a constant speed $V$. The lateral natural frequencies for a uniform fixed-fixed cable not on an elastic foundation can be determined from Eq. (26a) as

$$
\omega_{n}=\frac{n \pi}{\ell} \sqrt{\frac{P}{m}}, \quad n=1,2,3, \ldots, N
$$

where $P$ is a constant tensile force applied to the ends of the cable.

Note that when the uniform beam is very flexible (i.e., $I_{y} \rightarrow 0$ and $I_{z} \rightarrow 0$ ) but subjected to a large axial tensile force $P$ appled at its ends, the beam can be considered as a uniform fixed-fixed cable and Eq. (26a) yields Eq. (32) provided $\gamma_{y}=\gamma_{z}=0$. The critical speed of a fluid flowing along a uniforn: fixed-fixed cable not on an elastic foundation is obtained by substituting Eq. (32) into Eq. (26) and results in Fq. (33).

$$
V_{c r}=\sqrt{\frac{1}{\alpha} \frac{P}{m}}
$$

where $\alpha$ is not zero. Equation (33) can be nondimensionalized with respect to the wave speed in the cable by dividing $V_{c r}$ by $(P / m)^{1 / 2}$. That is,

$$
\beta_{c r}=\sqrt{\frac{1}{\alpha}}
$$

The critical speed of a fluid flowing along a uniform fixed-fixed cable supported on a uniform elastic foundation is given by

$$
V_{c r}=\sqrt{\frac{P}{m} \frac{1}{\alpha}\left[1+\frac{k_{y}}{P(\pi / \ell)^{2}}\right]} .
$$

The inequality (27) is not applicable. For this case the minimum critical speed $V_{c r}$ always occurs when $N=1$. The critical speed ratio of the fluid is expressed by

$$
\beta_{c r}=\sqrt{\frac{1}{\alpha}\left[1+\frac{k_{y}}{P(\pi / \ell)^{2}}\right]} .
$$




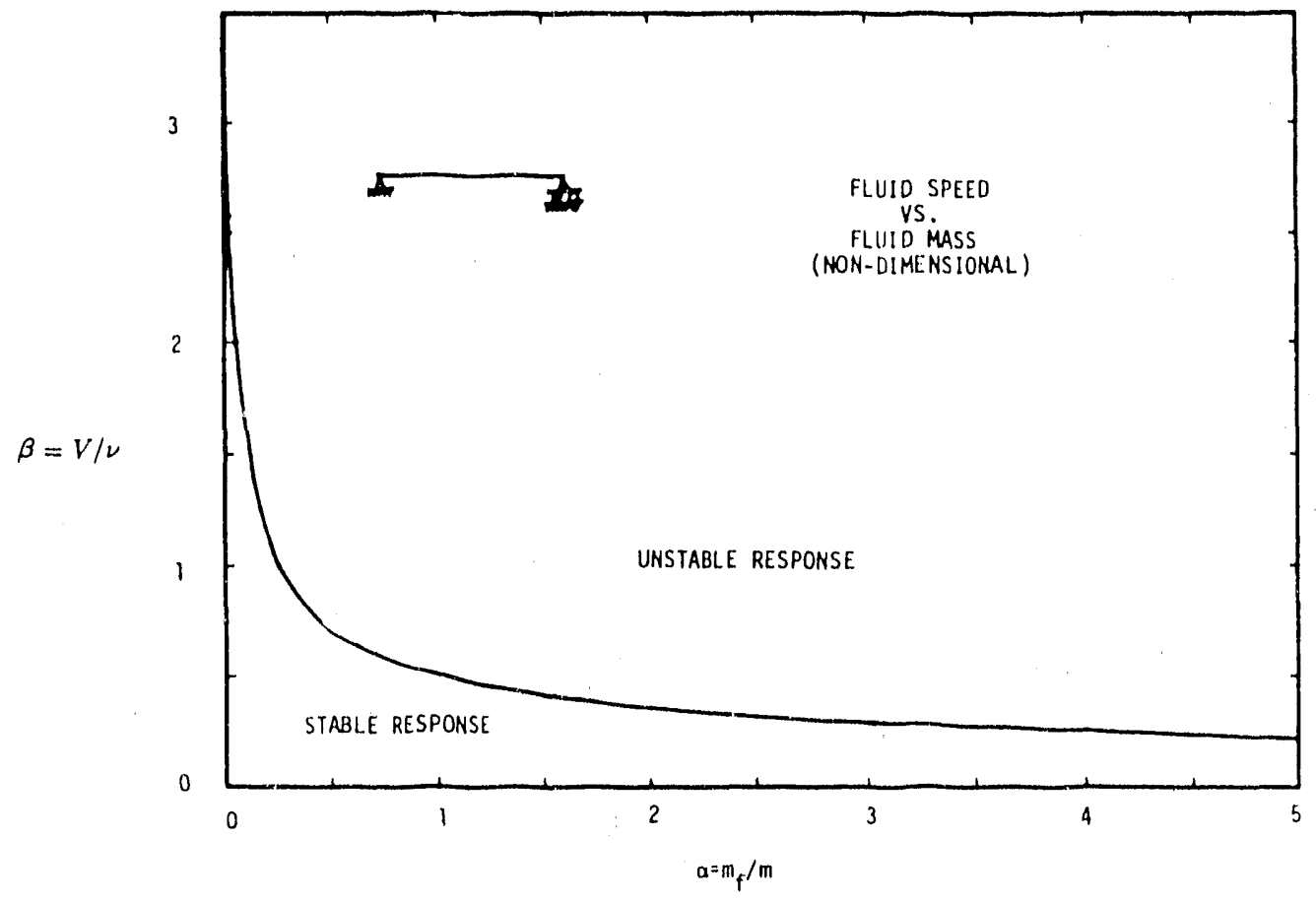

Figure 2. Regions of stable and unstable lateral response for system shown in Figure 1 for $P_{y}^{*}=\gamma_{y}=\omega_{x}=0$.

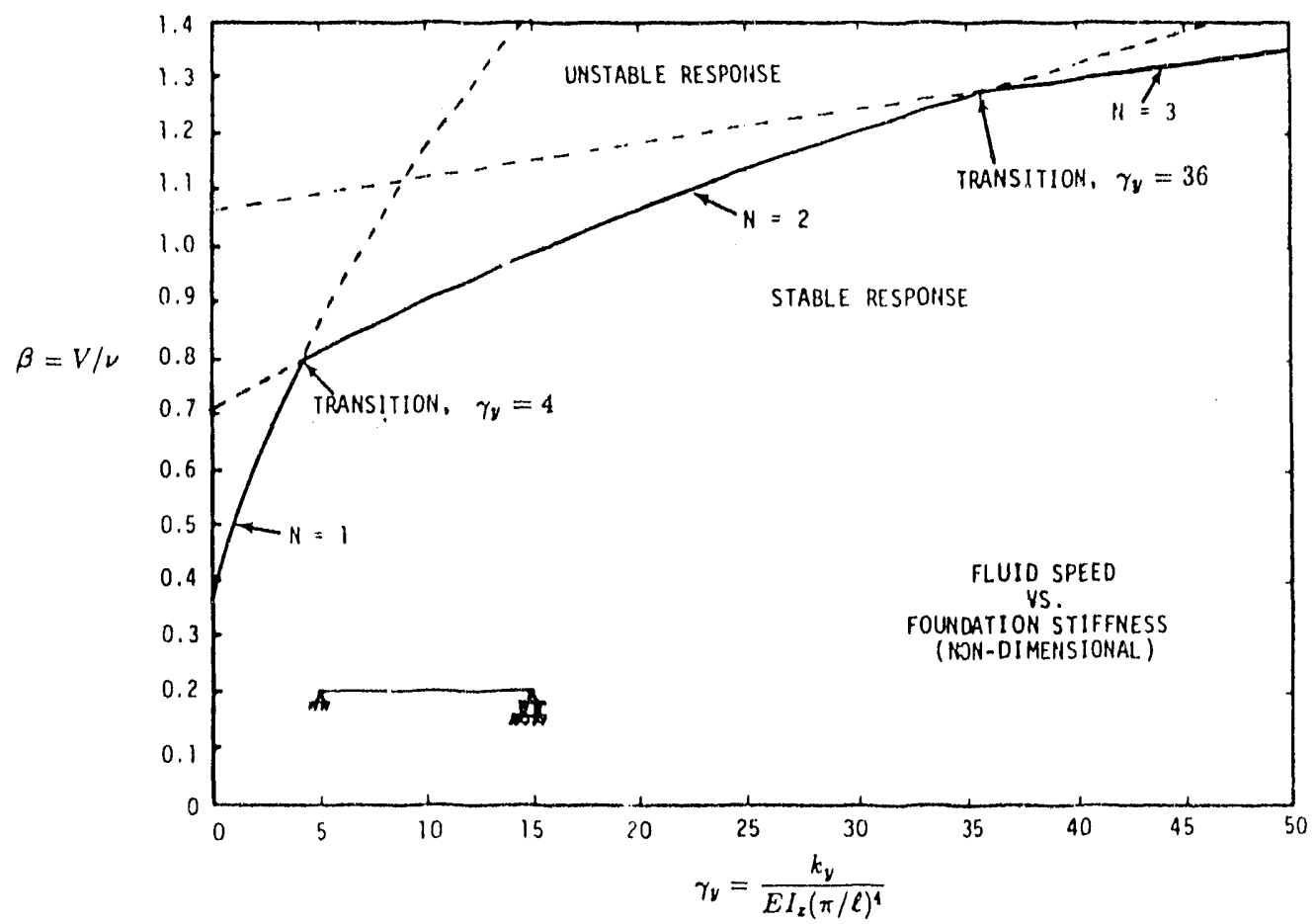

Figure 3. Regions of stable and unstable lateral response for system shown in Figure 1 for $P_{y}^{*}=\omega_{x}=0$ and $\alpha=2$. The solid line separates the regions of stable and unstable response. 


\section{Resonance for a Rotating Beam or Rotating Cable with Unequal Lateral Stiffness in the $y$ and 2 Directions}

When the dan ping factors, $\xi_{N y}$ and $\xi_{N z}$, are zero and the terms involving the rotary inertia and the ch.unge in direction of the angular momentum for an element of beam cross-section are neglected, the simplified equations of motion for the $N^{\text {th }}$ generalized beam coordinates, Eqs. (23) and (24), reduce to

$$
\begin{aligned}
& \ddot{f}_{N}(t)+\left[\frac{\omega_{N_{y}}^{2}-\alpha(N \pi / \ell)^{2} V^{2}}{(1+\alpha)}-\omega_{x}^{2}\right] f_{N}(t)-2 \omega_{x} \dot{q}_{N}(t)=0 \\
& \ddot{q}_{N}(t)+\left[\frac{\omega_{N z}^{2}-\alpha(N \pi / \ell)^{2} V^{2}}{(1+\alpha)}-\omega_{x}^{2}\right] q_{N}(t)+2 \omega_{x} \dot{f}_{N}(t)=0
\end{aligned}
$$

where

$$
\begin{aligned}
\omega_{N y}^{2} & =\frac{E I_{z}}{m}\left(\frac{\pi}{\ell}\right)^{4}\left[N^{4}+N^{2} \frac{P}{P_{e y}}+\gamma_{y}\right] \\
P_{e y} & =E I_{z}\left(\frac{\pi}{\ell}\right)^{2} \\
\gamma_{y} & =\frac{k_{y}}{E I_{z}(\pi / \ell)^{4}} \\
\omega_{N z}^{2} & =\frac{E I_{y}}{m}\left(\frac{\pi}{\ell}\right)^{4}\left[N^{4}+N^{2} \frac{P}{P_{e z}}+\gamma_{z}\right] \\
P_{e z} & =E I_{y}\left(\frac{\pi}{\ell}\right)^{2} \\
\gamma_{z} & =\frac{k_{z}}{E I_{y}(\pi / \ell)^{4}}
\end{aligned}
$$

Here $\omega_{N y}$ and $\omega_{N z}$ are the undamped natural bending frequencies associated with iateral motion of the beam due to bending vibration in the $y$ and $z$ directions, respectively, when the beam is not spinning (i.e., $\omega_{x}=0$ ) and the fluid mass per unit length, $m_{f}$, is zero. $P_{e y}$ and $P_{e z}$ are the magnitudes of the Euler buckling loads in the $y$ and $z \mathrm{di}-$ rections, respectively. $k_{y}$ and $k_{z}$ are the foundation stiffnesses per unit length in the $y$ and $z$ directions, respectively. $I_{y}$ and $I_{z}$ are the centroidal area moments of inertia of the beam cross-section about the $y$ and $z$ axes, respectively.

$f_{N}(t)$ and $q_{N}(t)$ are expressed by

$$
\begin{aligned}
& f_{N}(t)=A_{N} e^{\lambda_{N} t} \\
& q_{N}(t)=B_{N} e^{\lambda_{N} t}
\end{aligned}
$$


Substituting Eqs. (36) and (37) into Eqs. (34) and (35) and setting the determinant of the coefficients equal to zero yields the characteristic equation for the spinning beam (i.e., $\left.\omega_{x} \neq 0\right)$ :

$$
\lambda_{N}^{4}+\left[\left(\Omega_{N y}^{2}-\omega_{x}^{2}\right)+\left(\Omega_{N z}^{2}-\omega_{x}^{2}\right)+4 \omega_{x}^{2}\right] \lambda_{N}^{2}+\left(\Omega_{N y}^{2}-\omega_{x}^{2}\right)\left(\Omega_{N z}^{2}-\omega_{x}^{2}\right)=0
$$

Rearranging Eq. (38) results in

$$
\lambda_{N}^{4}+2\left[\frac{\left(\Omega_{N y}^{2}+\Omega_{N z}^{2}\right)}{2}+\omega_{x}^{2}\right] \lambda_{N}^{2}+\left(\Omega_{N y}^{2}-\omega_{x}^{2}\right)\left(\Omega_{N z}^{2}-\omega_{x}^{2}\right)=0
$$

where

$$
\Omega_{N y}^{2}=\frac{\omega_{N y}^{2}-\alpha\left(\frac{N \pi}{\ell}\right)^{2} V^{2}}{1+\alpha}
$$

and

$$
\Omega_{N z}^{2}=\frac{\omega_{N z}^{2}-\alpha\left(\frac{N \pi}{\ell}\right)^{2} V^{2}}{1+\alpha}
$$

The four roots to the characteristic Eq. (39) are given by

$$
\begin{gathered}
{ }_{1} \lambda_{N}=i\left\{C_{N}-D_{N}\right\}^{1 / 2} \quad \text { or } \quad{ }_{1} \lambda_{N}=i\left({ }_{1} \Omega_{N}^{\text {apparent }}\right)^{1 / 2} \\
{ }_{2} \lambda_{N}=-i\left\{C_{N}-D_{N}\right\}^{1 / 2} \quad \text { or }{ }_{2} \lambda_{N}=-i\left({ }_{1} \Omega_{N}^{\text {apparent }}\right)^{1 / 2} \\
{ }_{3} \lambda_{N}=i\left\{C_{N}+D_{N}\right\}^{1 / 2} \text { or }{ }_{3} \lambda_{N}=i\left({ }_{2} \Omega_{N}^{\text {apparent }}\right)^{1 / 2} \\
{ }_{4} \lambda_{N}=-i\left\{C_{N}+D_{N}\right\}^{1 / 2} \quad \text { or }{ }_{4} \lambda_{N}=-i\left({ }_{2} \Omega_{N}^{\text {apparent }}\right)^{1 / 2}
\end{gathered}
$$

where

$$
\begin{gathered}
C_{N}=\left[\left(\frac{\Omega_{N y}^{2}+\Omega_{N z}^{2}}{2}\right)+\omega_{x}^{2}\right] \\
D_{N}=\left[\left(\frac{\Omega_{N y}^{2}-\Omega_{N z}^{2}}{2}\right)^{2}+2 w_{x}^{2}\left(\Omega_{N y}^{2}+\Omega_{N z}^{2}\right)\right]^{1 / 2}
\end{gathered}
$$


and $N=1,2,3, \ldots, r$. Here $\Omega_{N y}$ and $\Omega_{N z}$ are the natural bending frequencies associated with lateral motion of the beam in the $y$ and $z$ directions, respectively, when the fluid is flowing along the beam (or pipe) with a constant soeed $V\left(V<V_{c r}\right)$ and the beam is not spinning (i.e., $\omega_{x}=0$ ).

Solving Eq. (39) for $\lambda_{N}$ results in the apparent tending natural frequencies associated with the free undamped lateral vibration for the spinning beam. These apparent frequencies are expressed by [iefer to Eqs. (39a), (39b), and (39c)]

$$
\begin{aligned}
& { }_{1} \Omega_{N}^{\text {apparent }}=\left\{\left[\left(\frac{\Omega_{N y}^{2}+\Omega_{N z}^{2}}{2}\right)+\omega_{x}^{2}\right]-\left[\left(\frac{\Omega_{N y}^{2}-\Omega_{N z}^{2}}{2}\right)^{2}+2 \omega_{x}^{2}\left(\Omega_{N y}^{2}+\Omega_{N z}^{2}\right)\right]^{1 / 2}\right\}^{1 / 2} \\
& { }_{2} \Omega_{N}^{\text {apparent }}=\left\{\left[\left(\frac{\Omega_{N y}^{2}+\Omega_{N z}^{2}}{2}\right)+\omega_{x}^{2}\right]+\left[\left(\frac{\Omega_{N y}^{2}-\Omega_{N z}^{2}}{2}\right)^{2}+2 \omega_{x}^{2}\left(\Omega_{N y}^{2}+\Omega_{N z}^{2}\right)\right]^{1 / 2}\right\}^{1 / 2}
\end{aligned}
$$

where $N=1,2,3, \ldots, r$. Note that for each of the $N$ bending modes associated with undamped free lateral vibration for the spinning beam, the amplitudes of vibration in the rotating body fixed $x-y$ and $x-z$ planes consist of an amplitude modulated sinusoidal vibration where the amplitude is sinusoidally modulated by a combination of frequencies composed of $\omega_{x}, \Omega_{N y}$ and $\Omega_{N z}$. Consequently, the free bending vibration of the $N^{\text {th }}$ mode for the spinning beam appears to be composed of two apparent bending frequencies $\left({ }_{1} \Omega_{N}^{\text {apparent }}\right.$ and ${ }_{2} \Omega_{N}^{a p p a r e n t}$ ) and neither of these apparent frequencies corresponds to the $N^{\text {th }}$ natural bending frequency for the nonspinning, bearn.

Also, observe that the $2 N$ apparent frequencies consist of a difference and a sum of "two frequencies" [refer to Eqs. (42) and (43)] and that these "two frequencies" are not as simple as those given by Eqs. (46) and (47). That is, the "two frequencies" given by Eqs. (42) and (43) result from a combination of the natural frequencies for the beam [i.e., $\Omega_{N y}$ and $\Omega_{N z}$; refer to Eqs. (40) and (41)] and the spin frequency $\omega_{x}$ of the beam.

For each mode of vibration, resonance occurs at two spin frequencies or critical spin speeds. That is,

$$
N \omega_{x \text { cr } 1}=\Omega_{N y}
$$

or

$$
N \omega_{x(r)}=\left[\frac{\omega_{N y}^{2}-\alpha(N \pi / \ell)^{2} V^{2}}{1+\alpha}\right]^{1 / 2}
$$

and

$$
{ }^{\prime} \omega_{x \text { cr } 2}=\Omega_{N z}
$$

or 


$$
{ }_{N} \omega_{x \mathrm{cr} 2}=\left[\frac{\omega_{N z}^{2}-\alpha(N \pi / \ell)^{2} V^{2}}{1+\alpha}\right]^{1 / 2}
$$

where $N=1,2,3, \ldots, r$. Equations (44) and (45) result from Eq. (42) when ${ }_{1} \Omega_{N}^{\text {apparent }}$ is zero.

In general, it is not expected that the critical spin speeds $\left({ }_{N} \omega_{x c r} i, i=1,2\right)$ will equal the beam natural bending frequencies associated with zero angular speed; that is Eqs. (44) and (45). For example, consider a cantilevered beam with a large disk at,tached to its free end. When the beam is spinning about its undeformed longitudinal axis, the gya uscopic effects of the disk (and to a much lesser extent the beam cross-sections) are expected, for the most part, to stiffen the beam resulting in critical speeds $\left({ }_{N} \omega_{x c r i}\right)$ which are, in general, higher than the natural frequencies $\left(\Omega_{N y}\right.$ and $\left.\Omega_{N z}\right)$ for the cantilevered beam.

Returning to Eqs. (42) and (43), note that when $\Omega_{N y}=\Omega_{N z}=\Omega_{N}$ (or $\omega_{N y}=$ $\omega_{N z}=\omega_{N}$; e.g., a beam with circular cross-section resting on a uniform anu equal elastic foundaiio:.1), Eqs. (42) and (43) reduce to Eqs. (46) and (47), respectively.

$$
\begin{aligned}
& { }_{1} \Omega_{N}^{\text {apparent }}=\Omega_{N}-\omega_{x} \\
& { }_{2} \Omega_{N}^{\text {apparent }}=\Omega_{N}+\omega_{x}
\end{aligned}
$$

with $N=1,2,3, \ldots, r$, where $\Omega_{N}$ is given by either Eq. (40) or (41) and $\omega_{N y}$ or $\omega_{N z}$ is given by either Eq. (34a) or (35a). Also, when $\omega_{x}=0$, the apparent frequencies equal the natural frequency, $\omega_{N}$; the amplitude of vibration is constant, and only one natural frequency is associated with each mode of vibration.

Consider Eqs. (46) and (47). When the spin speed of the beam, $\omega_{x}$, is zero, the apparent frequencies are identical for lateral vibration of the $N^{\text {th }}$ mode in the $y$ and $z$ directions (i.e., ${ }_{1} \Omega_{N}^{\text {apparent }}={ }_{2} \Omega_{N}^{\text {apparent }}=\Omega_{N}$ ). As $\omega_{x}$ is gradually increased, ${ }_{1} \Omega_{N}^{\text {apparent }}$ decreases by $\omega_{x}$ and ${ }_{2} \Omega_{N}^{a p p a r e n t}$ increases by $\omega_{x}$. When $\omega_{x}$ eventually becomes equal to $\Omega_{N}$ (i.e., ${ }_{1} \Omega_{N}^{\text {apparent }}=0$ ) resonance occurs and the amplitudes of vibration of the $N^{\text {th }}$ mode increase linearly with time and the resonant frequency of vibration for the $N^{\text {th }}$ mode becomes equal to $2 \Omega_{N}$. That is, ${ }_{1} \Omega_{N}^{\text {apparent }}=0$ and ${ }_{2} \Omega_{N}^{\text {apparent }}=2 \Omega_{N}$ at resonance. Note that in this case $N \omega_{x c r}=\Omega_{N}$ where $N=1,2,3, \ldots, r$.

Now consider Eqs. (42) and (43). When the spin speed of the beam, $\omega_{x}$, is zero, the apparent frequencies for lateral vibration of the $N^{\text {th }}$ mode in the $y$ and $z$ directions are $\Omega_{N y}$ and $\Omega_{N z}$, respectively. As $\omega_{x}$ is gradually increased and approaches $\Omega_{N y}$, resonance occurs and the resonant frequency of vibration for the $N^{\text {th }}$ mode, in the $y$ and $z$ directions, equals $\sqrt{3 \Omega_{N y}^{2}+\Omega_{N z}^{2}}$. As $\omega_{x}$ is increased further and approaches $\Omega_{N z}$, a second resonance occurs for the $N^{\text {th }}$ mode and the resonant frequency of vibration for the $N^{\text {th }}$ mode in the $y$ and $z$ directions, associated with the second resonance, equals $\sqrt{\Omega_{N y}^{2}+3 \Omega_{N z}^{2}}$ [refer to Eqs. (63) and (64)]. (Note that in the second paragraph of the Results section, it is assumed that $\omega_{N y}<\omega_{N z}$ and that this is an arbitrary choice.) 
Case 1: Fluid Mass is Zero-When the fluid mass per unit length, $m_{f}$, is zero (i.e., $\alpha=0$ ), resonance occurs when the angular spin speed of the beam (or tube), $\omega_{x}$, equals any of the $2 N$ natural bending frequencies, $\omega_{N y}$ and $\omega_{N z}$. That is, re:'onance occurs when

$$
N \omega_{x \operatorname{cr} 1}=\omega_{N y}
$$

and

$$
N \omega_{x \text { cr } 2}=\omega_{N z}
$$

and $N=1,2,3, \ldots, r$.

Case 2: Fluid Speed is Zero- - $\mathrm{V}$ hen the fluid speed $V$ along the tube equals zero but the tube or pipe is filled with a fluid of mass $m_{f}$ per unit length (i.e., $\alpha \neq 0$ ), resonance occurs when

$$
N \omega_{x c r 1}=\frac{\omega_{N_{\tilde{z}}}}{\sqrt{1+\alpha}}
$$

and

$$
N \omega_{x c r 2}=\frac{\omega_{N z}}{\sqrt{1+\alpha}}
$$

and $N=1,2,3, \ldots, r$.

Case 3: Fluid Spead is Less Than the Critical Fluid Speed-When the fluid speed $V$ is less than the critical fluid speed $V_{c r}$ (i.e., $\Omega_{N y}^{2}>0$ in Eq. (40); recall the assumption that $\left.\omega_{N y}<\omega_{N z}\right)$, resonance occurs when

$$
N \omega_{x c r 1}=\sqrt{\frac{\omega_{N y}^{2}-\alpha(N \pi / \ell)^{2} V^{2}}{1+\alpha}}
$$

and

$$
N \omega_{x c r 2}=\sqrt{\frac{\omega_{N z}^{2}-\alpha(N \pi / \ell)^{2} V^{2}}{1+\alpha}}
$$

and $N=1,2,3, \ldots, r$. In this case, if the fluid speed $V$ is high enough, resonance can occur at very low values of angular speed, $\omega_{x}$.

Case 4: Fluid Speed Equals the Critical Fluid Speed-When the fluid speed $V$ equals the critical fluid speed $V_{c r}$ (i.e., $\Omega_{N y}=0$ ), resonance occurs. That is, when $V=V_{c r}$, where

$$
V_{c r}=\frac{\ell}{N \pi} \omega_{N y} \sqrt{\frac{1}{\alpha}}
$$


and the inequality (27) applies, resonance occurs when

$$
{ }_{N} \omega_{x \text { cr } 1}=0
$$

and

$$
N \omega_{x c r 2}=\Omega_{N z}
$$

where

$$
\Omega_{N z}=\sqrt{\frac{\omega_{N z}^{2}-\omega_{N y}^{2}}{1+\alpha}}
$$

and $N=1,2,3, \ldots, r$. When the beam bending natural frequencies (or the tube lateral natural frequencies) are identical in the $y$ and $z$ directions (i.e., $\omega_{N y}=\omega_{N z}$ ), resonance occurs for any spin speed, $\omega_{x}$, greater than zero.

Case 5: Fluid Speed Exceeds the Critical Fluid Speed-When the fluid speed $V$ along the tube exceeds the critical fluid speed $V_{c r}$ (i.e., $\Omega_{N y}^{2}$ is negative), dynamic instability occurs. That is, when $V>V_{c r}$, where

$$
V_{c r}=\frac{\ell}{N \pi} \omega_{N y} \sqrt{\frac{1}{\alpha}}
$$

and the inequality (27) applies, resonance occurs for any spin speed $\omega_{x}$ greater than zero. However, if the spin speed $\omega_{x}$ is zero, a dynamic instability occurs which is analogous to static buckling (divergence) when $V=V_{c r}$. Consequently, for this case there are no oscillatory lateral motions associated with the dynamic instability and the critical speed for the fluid is independent of the viscous damping factor for the beam and foundation.

In Eqs. (48) through (58), $\omega_{N y}$ and $\omega_{N z}$ for the beam shown in Figure 1 are given by

$$
\begin{aligned}
\omega_{N y}^{2} & =\frac{E I_{z}}{m}\left(\frac{\pi}{\ell}\right)^{4}\left[N^{4}+N^{2} \frac{P}{P_{e y}}+\gamma_{y}\right] \\
P_{e y} & =E I_{z}\left(\frac{\pi}{\ell}\right)^{2} \\
\gamma_{y} & =\frac{k_{y}}{E I_{z}(\pi / \ell)^{4}}
\end{aligned}
$$




$$
\begin{aligned}
\dot{\omega}_{N z}^{2} & =\frac{E I_{y}}{m}\left(\frac{\pi}{\ell}\right)^{4}\left[N^{4}+N^{2} \frac{P}{P_{e z}}+\gamma_{z}\right] \\
P_{e z} & =E I_{y}\left(\frac{\pi}{\ell}\right)^{2} \\
\gamma_{z} & =\frac{k_{z}}{E I_{y}(\pi / \ell)^{4}}
\end{aligned}
$$

Recall the assumption that $\omega_{N_{y}}<\omega_{N_{z}}$. Therefore, for the cable, it is assumed that $k_{y}<k_{z}$. For a uniform fixed-fixed cable, $\omega_{N_{y}}$ and $\omega_{N z}$ are given by

$$
\omega_{N_{y}}=N \frac{\pi}{\ell} \sqrt{\frac{P}{m}\left[1+\frac{k_{y}}{N^{2} P(\pi / \ell)^{2}}\right]}
$$

and

$$
N_{y z}=N \frac{\pi}{\ell} \sqrt{\frac{P}{m}\left[1+\frac{k_{z}}{N^{2} P(\pi / \ell)^{2}}\right]}
$$

and $N=1,2,3 \ldots, r$.

In Eqs. (61) and (62) $\mathrm{P}$ is a constant axial tensile force applued to the ends of the cable and $m$ is the cable mass per unit length. Use of Eqs. (61) and (62) in Eqs. (48) through (5T) will give the critical spir speeds, $N \omega_{z}$ crs, which result in resonance for a rotating, uniform, fixed-fixed cable supported on an elastic foundation and conveying an incompressibie, frictionless fluid moring at a constant speed $1^{\circ}$. For a cable the critical fluid speed occurs when $N=1$ refer to Eq. (33b). and the inequality (27) is not applicable.

Frequency of Lateral Vibration at Resonance-For each mode of undamped lateral vibration. resonance can occur at two critical spin speeds provided the fluid speed is less than or equal to the minimum crit:cal fluid speed $V_{c r}$. The amplitude of lateral vibration increases linearly with respect to time and the frequency of lateral vibration for the $N^{\text {th }}$ mode at resonance is determined from equations (63) or (64); that is, Case A or Case B. Note that the iwo apparent resonant frequencies for the $N^{\text {th }}$ mode of lateral vibration consist of a certain combination of the two critical spin speeds associated with resonance for the $N^{\text {th }}$ mode of vibration. (Refer to Cases 1 through 3 for the critical speeds of interest.)

Case A: Spin Speed Equal to First Critical Speed for . Th $^{\text {th }}$ Mode.

$$
N_{\text {Nut }}=\Omega_{N_{y}} \quad \text { (i.e., }, \Omega_{N}^{\text {appurent }}=0 \text { ) }
$$

Frequency of lateral vibration at resonance:

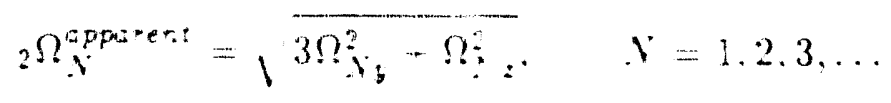




$$
{ }_{2} \Omega_{N}^{\text {apparent }}=\sqrt{3\left(N \omega_{x \mathrm{cr} 1}^{2}\right)+N \omega_{x \mathrm{cr} \text { i }}^{2}}
$$

Case B: Spin Speed Equal to Second Critical Speed for $N^{\text {th }}$ Mote.

$$
\left.N \omega_{x \text { cr } 2}=\Omega_{N z} \quad \text { (i.e., } 1 \Omega_{N}^{\text {apparent }}=0\right)
$$

Frequency of lateral vibration at resonance.

$$
{ }_{2} \Omega_{N}^{\text {apparent }}=\sqrt{\Omega_{N y}^{2}+3 \Omega_{N z}^{2}}, \quad N=1,2,3, \ldots
$$

or

$$
{ }_{2} \Omega_{N}^{\text {apparent }}=\sqrt{N \omega_{x \mathrm{cr} 1}^{2}+3\left({ }_{N} \omega_{x \mathrm{cr} 2}^{2}\right)}
$$

$\Omega_{N y}$ and $\Omega_{N z}$ are given by Eqs. (40) and (41). For Case A or B, the $2 N$ critical spin speeds of interest, $N \omega_{x c r i}(i=1,2)$, are given in Cases 1 through 3 . Note that $\omega_{N y}$ and $\omega_{N z}$ are determined by Eqs. (59) and (60) for a beam and by Eqs. (61) and (62) for a cable.

When $\Omega_{N y}=\Omega_{N z}=\Omega_{N}$ (or $\omega_{N y}=\omega_{N z}=\omega_{N}$; e.g., a beam with a circular cross-section resting on a uniform and equal elastic foundation), the apparent frequency of lateral vibration at resonance for Cases $A$ and $B$ is equal to $2 \Omega_{N}$. That is,

$$
{ }_{2} \Omega_{N}^{\text {apparent }}=2 \Omega_{N}
$$

or

$$
{ }_{2} \Omega_{N}^{\text {apparent }}=2 \sqrt{\frac{\omega_{N}^{2}-\alpha(N \pi / \ell)^{2} V^{2}}{1+\alpha}}
$$

where

$$
\begin{gathered}
\omega_{N}^{2}=\frac{E I}{m}\left(\frac{\pi}{\ell}\right)^{4}\left[N^{4}+N^{2} \frac{P}{P_{e}}+\gamma\right] \\
P_{e}=E I\left(\frac{\pi}{\ell}\right)^{2} \\
\gamma=\frac{k}{E I(\pi / \ell)^{4}}
\end{gathered}
$$

and $N=1.2,3, \ldots, r$.

Equations (66) and (67) can be simplified for application to a cable. 


\section{Comparison Between Experimental Measurernents and Predicted Values}

To illustrate the use of Eqs. (46) and (47), consider the foilowing brief description of a field experiment and some associated measured resuits. For additional information, refer to $[2]$.

Figures 4 and 5 illustrate an 8 -inch-diameter test projectile and the usual gun firing environments. Figure 6 is an enlarged view of the forward beam within the test projectile. The cylindrical disk on the end of the beam is used to tune the beam to a specified natural frequency associated with the fi:st mode of vibration due to bending.

As the gun-fired test projectile travels along the rifled gun tube toward the muzzle, the beam and disk are subjected to an axial acceleration $\ddot{U}$, an angular acceleration $\dot{\omega}_{x}$, and an angular velocity $\omega_{x}$, which results in a nearly quasi-static response of the beam.

Almost immediately upon barrel exit the character of beam response changes suddenly from quasi-static to dynamic (refer to Figure 7). Note that the beam vibration shown in Figure 7 occurs in the body fixed $x-y$ plane. Due to the Coriolis effect an analogous vibration also occurs in th body fixed $x-z$ plane. The vibration which begins at barrel exit is primarily caused by the sudden reduction (in about 0.2 milliseconds) of pressure acting on the projectile base. When this occurs, the rigid body axial inertia loads acting on the beam and disk suddenly decrease to zero (drag is neglected) and the bending moment in the beam at the strain gage location changes almost instantaneously relative to the fundamental period for lateral oscillation. Consequently, for the forward beam, the sudden increase in the bending moment at the location of the strain gage ( $358.5^{\circ}$ and 1 inch from its fixed end) results in additional compressive strain in the beain and its subsequent dynamir response.

The wave shape subsequent to barrel exit is composed of two apparent bending frequencies, each associated with the first nıode for lateral vibration. Figure 8 shows the measured and predicted values for these frequencies. The first natural bending frequency for the beam, when it is not spinning, is $393 \mathrm{~Hz}$ (refer to Appendix $\mathrm{G}$ in [2]) and the spin frequency of the projectile is $183 \mathrm{~Hz}$. Therefore, the two calculated apparent bend. ing frequencies for the first mode are $210 \mathrm{~Hz}$ and $576 \mathrm{~Hz}$. The measured frequencies are $213 \mathrm{~Hz}$ and $575 \mathrm{~Hz}$. The agreement between the measured and calculated frequencies, using Eqs. (46) and (47), is quite good.

In applying Eqs. (46) and (47) to the beam shown $j_{a}$ Figure 6, note that there is no fluid present (i.e., $\alpha=0$ ) and that $\Omega_{N y}=\Omega_{N_{z}}=\omega_{N_{y}}=\omega_{N_{z}}=\omega_{N}$ where $\mathrm{N}=1$.

When there is no axial load acting on the beam $(P=0)$ and the foundation stiffness is zero $(\gamma=0)$, the natural bending frequencies for the beam shown in Figure 6 are given by

$$
\omega_{i}=\frac{\beta_{i}}{\ell^{2}} \sqrt{\frac{E I}{m}}, \quad i=1,2, \ldots, N
$$

and $\beta_{i}$ is determined from 


$$
\begin{aligned}
& 1+b^{2} \alpha^{2} \beta^{4}+\left\{-\alpha \beta^{3}\left(a^{2}+b^{2}\right)-\alpha \beta+\frac{\beta}{S}\left[\alpha^{2} \beta^{4}\left(a^{2}+b^{2}\right)-\left(1+a \alpha \beta^{2}\right)^{2}\right]\right\} \sin \beta \cosh \beta \\
& -2\left[a \alpha \beta^{2}+\frac{\alpha \beta^{2}}{S}\right] \sin \beta \sinh \beta+\left[1-\alpha^{2} \beta^{4} b^{2}-\frac{2 \alpha \beta^{4}}{S}\left(a^{2}+b^{2}\right)\right] \cos \beta \cosh \beta \\
& +\left\{\alpha \beta-\alpha \beta^{3}\left(a^{2}+b^{2}\right)+\frac{\beta}{S}\left[\left(1-a \alpha \beta^{2}\right)^{2}-\alpha^{2} \beta^{4}\left(a^{2}+b^{2}\right)\right]\right\} \cos \beta \sinh \beta=0
\end{aligned}
$$

where

$$
\begin{aligned}
a & =\frac{\epsilon}{\ell} \\
b^{2} & =\frac{J_{D}}{M_{D} \ell^{2}} \\
\alpha & =\frac{M_{D}}{m \ell} \\
S & =\frac{k \ell}{E I}
\end{aligned}
$$

The following definitions apply for the terms in Eq. (70).

$\epsilon$ is the distance from the end of the beam to the mass center of a rigid disk attached to the free end of a beam.

$J_{D}$ is the mass moment of inertia of a rigid disk about the $y$ or $z$ axis originating at the mass center of a disk.

$M_{D}$ is the mass of a rigid disk.

$k$ is the bending spring constant for a spring located at the "fixed" end (i.e., at $x=0$ ) of a beam.

The symbols used in Eqs. (68) and (69) are also defined in [2].

Equation (69) represents the characteristic equation for the Euler beam shown in Figure 6 when the beam is not spinning (i.e., $\omega_{x}=0$ ). For a specified set of parameters (i.e., $a, b, \alpha$, and $S$ ), Eq. (69) will yield $N$ roots $\beta_{i}$ and, consequently, $N$ natural bending frequencies for the beam.

Laboratory experiments on test beams indicate Eq. (69) accurately predicts the natural frequencies for lateral vibration. Two beams were tested and the results are summarized in Tables II and III.

If the modulus of elasticity, E, is $28 \times 10^{6} \mathrm{lb} /$ in. ${ }^{2}$ rather than $30 \times 10^{6} \mathrm{lb} /$ in. ${ }^{2}$, the calculated natural frequencies are reduced by $3.39 \%$. Consequently, some of the differences listed in the fourth column in Tables II and III could be due to material property variations.

Equation (69) is applicable for other boundary conditions: for example, a cantilever beam without a disk attacned to its free end. For this case 


$$
\begin{aligned}
& \alpha=\frac{M_{D}}{m \ell}=0 \\
& S=\frac{k \ell}{E I} \rightarrow \infty
\end{aligned}
$$

and Eq. (69) reduces to

$$
1+\cos \beta \cosh \beta=0 \text {. }
$$

TABLE II.

RESULTS OF LABORATORY TESTS ON FIRST BEAM.

Beam parameters: $\ell=4.0 \mathrm{in}$., $D=0.40 \mathrm{in}$., $2 \epsilon=1.0 \mathrm{in}$., $D_{D}=1.20 \mathrm{in}$., $E=30 \times 10^{6} \mathrm{lb} /$ in. $^{2}$.

Nondimensional parameters: $a=\epsilon / \ell=0.125, b^{2}=J_{D} / M_{D} \ell^{2}=0.010833$, $\alpha=M_{D} / m \ell=2.25, S=2.2727 \times 10^{3}, k=21.42 \times 10^{6} \mathrm{in} . \mathrm{lb} / \mathrm{rad}$.

\begin{tabular}{cccc}
$\begin{array}{c}\text { Mode } \\
\text { No. }\end{array}$ & $\begin{array}{c}\text { Measured Natural } \\
\text { Frequency }(\mathrm{Hz})\end{array}$ & $\begin{array}{c}\text { Calculated Natural } \\
\text { Frequency }(\mathrm{Hz})\end{array}$ & $\begin{array}{c}\text { Percent } \\
\text { Difference }\end{array}$ \\
\hline 1 & 183.3 & 186.7 & 1.85 \\
2 & 1914. & 2005.4 & 4.78
\end{tabular}

TABLE III.

RESULTS OF LABORATORY TESTS ON SECOND BEAM.

Beam Parameters: $\ell=4.0$ in., $D=0.40$ in., $2 \epsilon=0.2$ in., $D_{D}=1.20$ in., $E=30 \times 10^{6} \mathrm{lb} /$ in. $^{2}$.

Nordimensional parameters: $a=\epsilon / \ell=0.025, b^{2}=J_{D} / M_{D} \ell^{2}=0.005833$, $\alpha=M_{D} / m \ell=0.45, S=2.2727 \times 10^{3}, k=21.42 \times 10^{6} \mathrm{in} .-\mathrm{lb} / \mathrm{rad}$.

\begin{tabular}{cccc}
$\begin{array}{c}\text { Mode } \\
\text { No. }\end{array}$ & $\begin{array}{c}\text { Measured Natural } \\
\text { Frequency }(\mathrm{Hz})\end{array}$ & $\begin{array}{c}\text { Calculated Natural } \\
\text { Frequency }(\mathrm{Hz})\end{array}$ & $\begin{array}{c}\text { Percent } \\
\text { Difference }\end{array}$ \\
\hline 1 & 393. & 408.7 & 3.99 \\
2 & 2925. & 3087.5 & 5.55
\end{tabular}




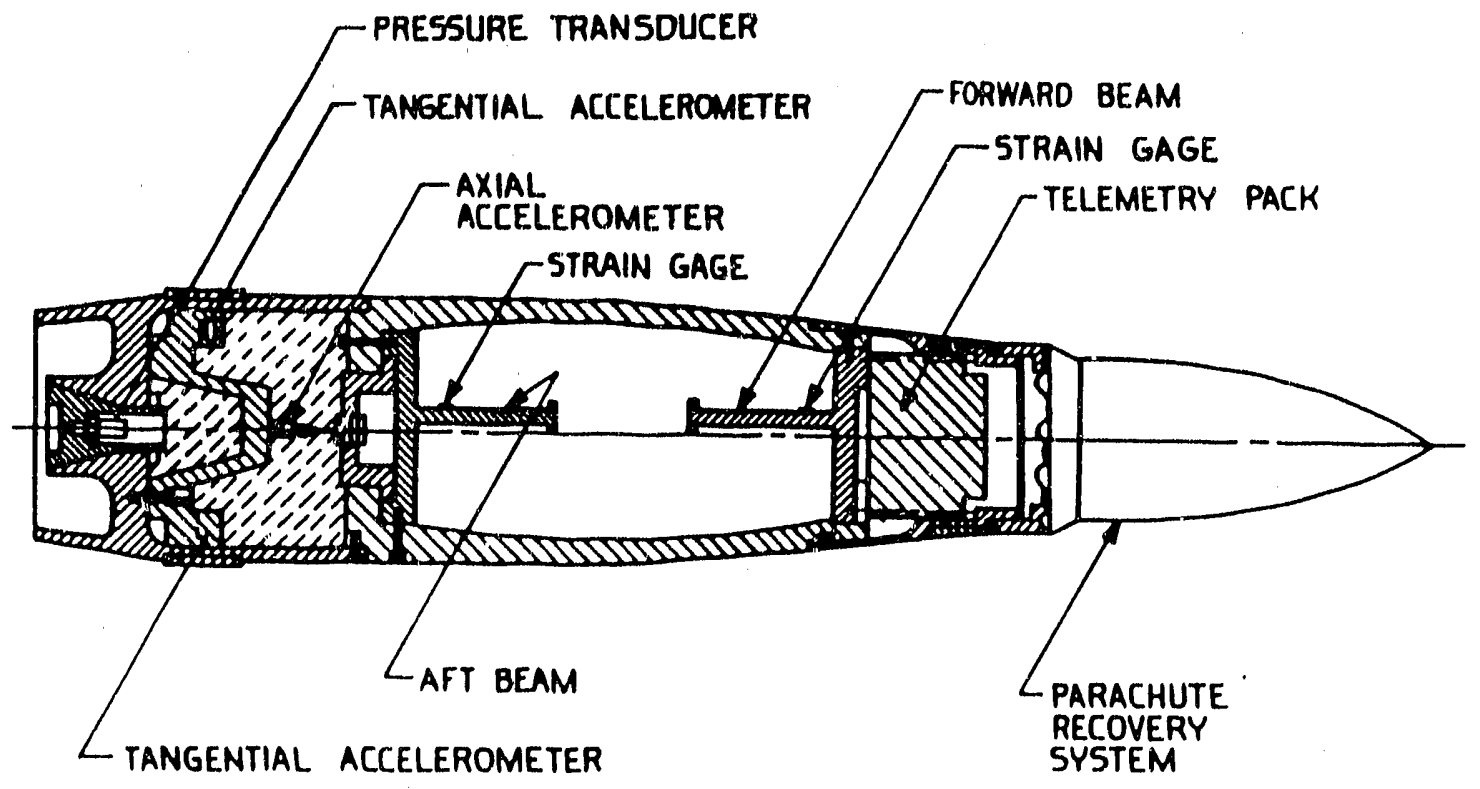

Figure 4. Eight-inch-diameter test projectile. 


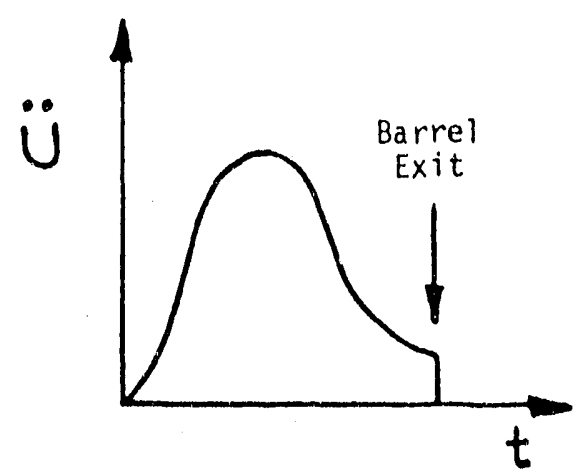

Figure 5a. Rigid body axial acceleration vs. time. Barrel exit time occurs at approximately 17.5 milliseconds. Maximum axial acceleration can approach 12,000 g's.

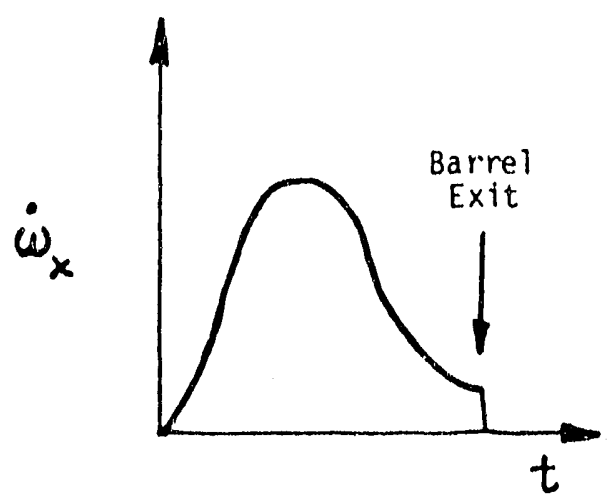

Figure 5b. Rigid body angular acceleration vs. time. Maximum angular acceleration can approach $182,000 \mathrm{rads} / \mathrm{sec}^{2}$.

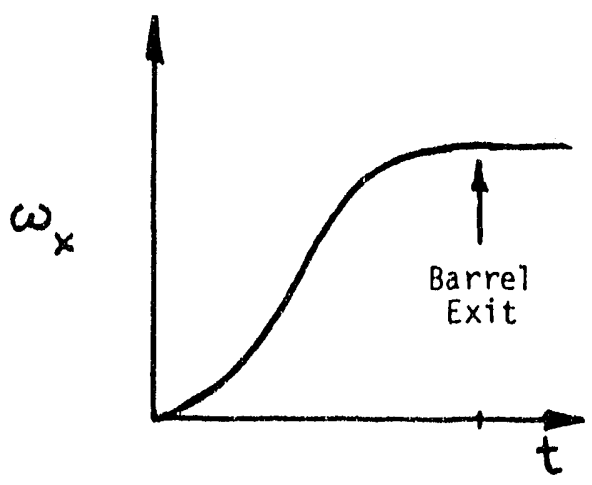

Figure 5c. Ricid body angular velocity vs. time. As the gun-fired test projectile travels along the ri gun tube toward the muzzle, the beam and disk are subjected to an axial acceleration $\dot{U}$, an angular acceleration $\dot{\omega}_{x}$, and an angular velocity $\omega_{x}$, which results in a nearly quasi-static response of the beam. Maximum angular velocity can approach $1,180 \mathrm{rads} / \mathrm{sec}(11,256 \mathrm{rpm})$. 


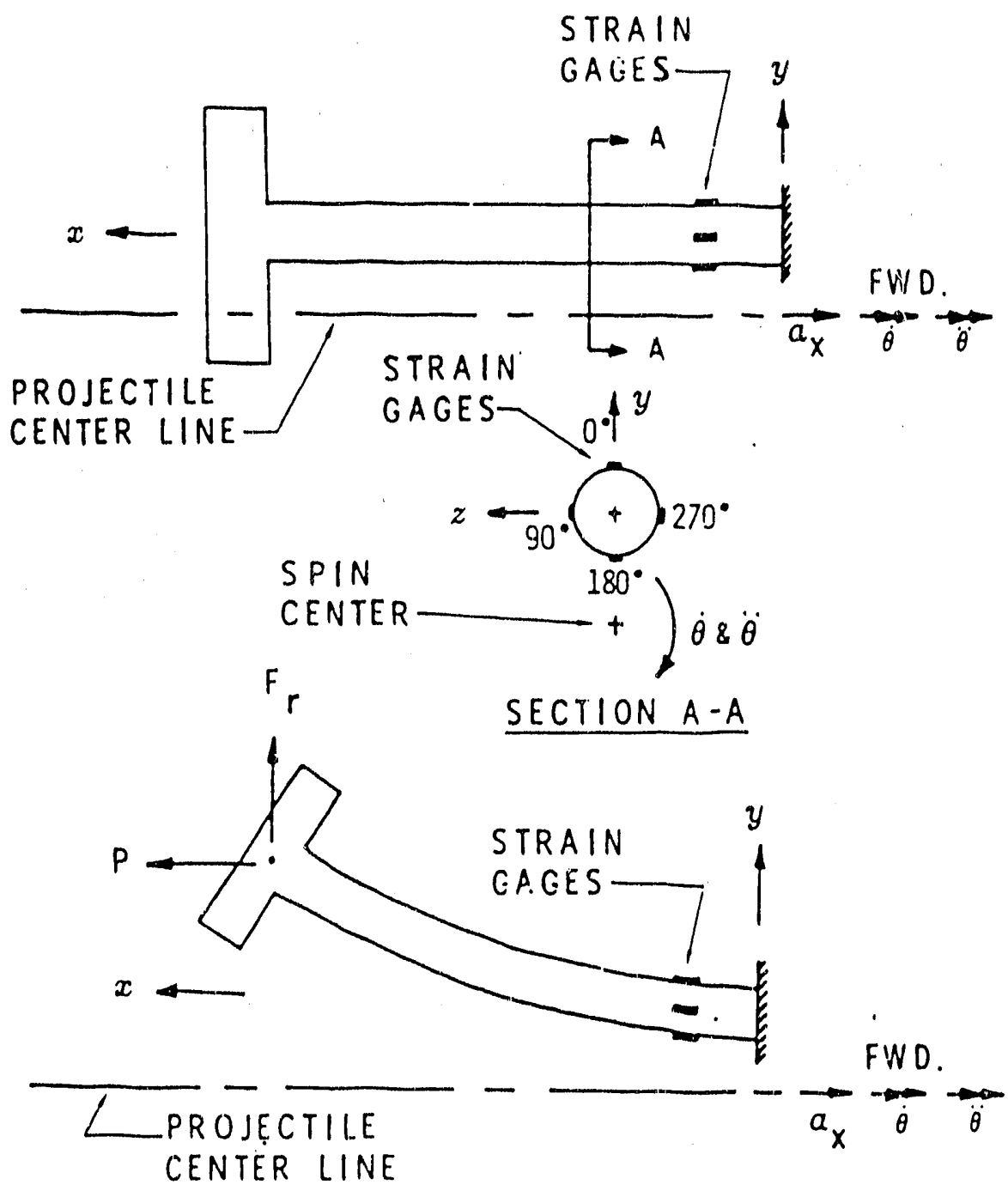

Figure 6. Forward beam (refer to Figure 4), illustrating the undeformed and deformed positions in the body-fixed $x-y$ plane. Note that for the forward beam $a_{x}=-\ddot{U}$, $\dot{\theta}=-\omega_{x}$, and $\ddot{\theta}=-\dot{\omega}_{x} . P$ and $F_{r}$ schematically represent the resultant axial and radial inertia forces acting at the center of mass of the disk. 


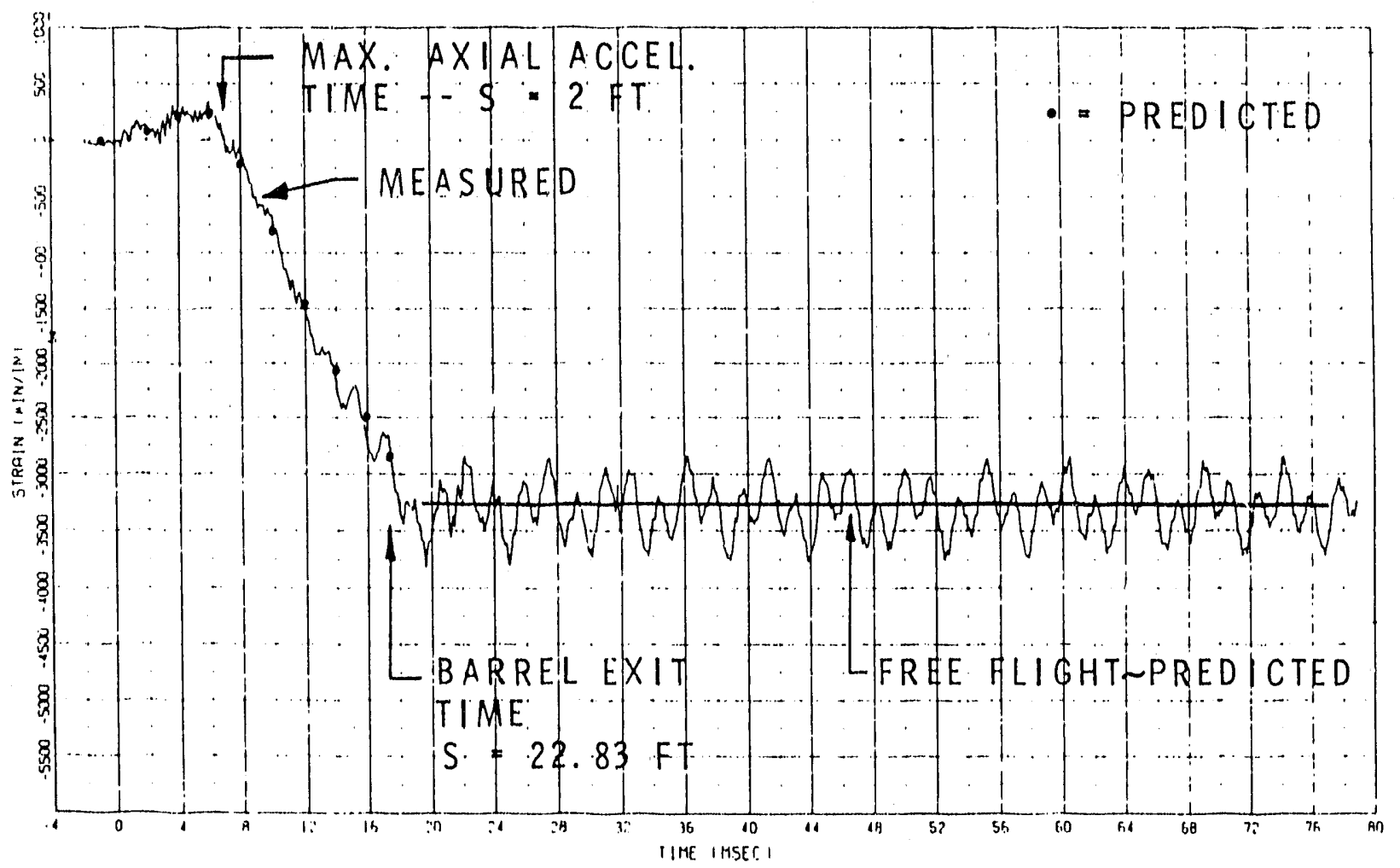

Figure 7. Measured strain-time history for a uniaxial strain gage located on the forward beam at $358.5^{\circ}$ and 1 inch from its fixed end. The test (GE021B) utilized essentially a new 8 -inch-diameter tube (90\% remaining life; tube number 34 ). The dots and heavy solid line represent predicted quasi-static strain values. The coupled lateral vibration subsequent to barrel exit results from the Coriolis effect. Note that the projectile exits the gun tube at approximately $17.5 \times 10^{-3} \mathrm{sec}$ and that the response of the beam prior to this time is essentially quasi-static. 


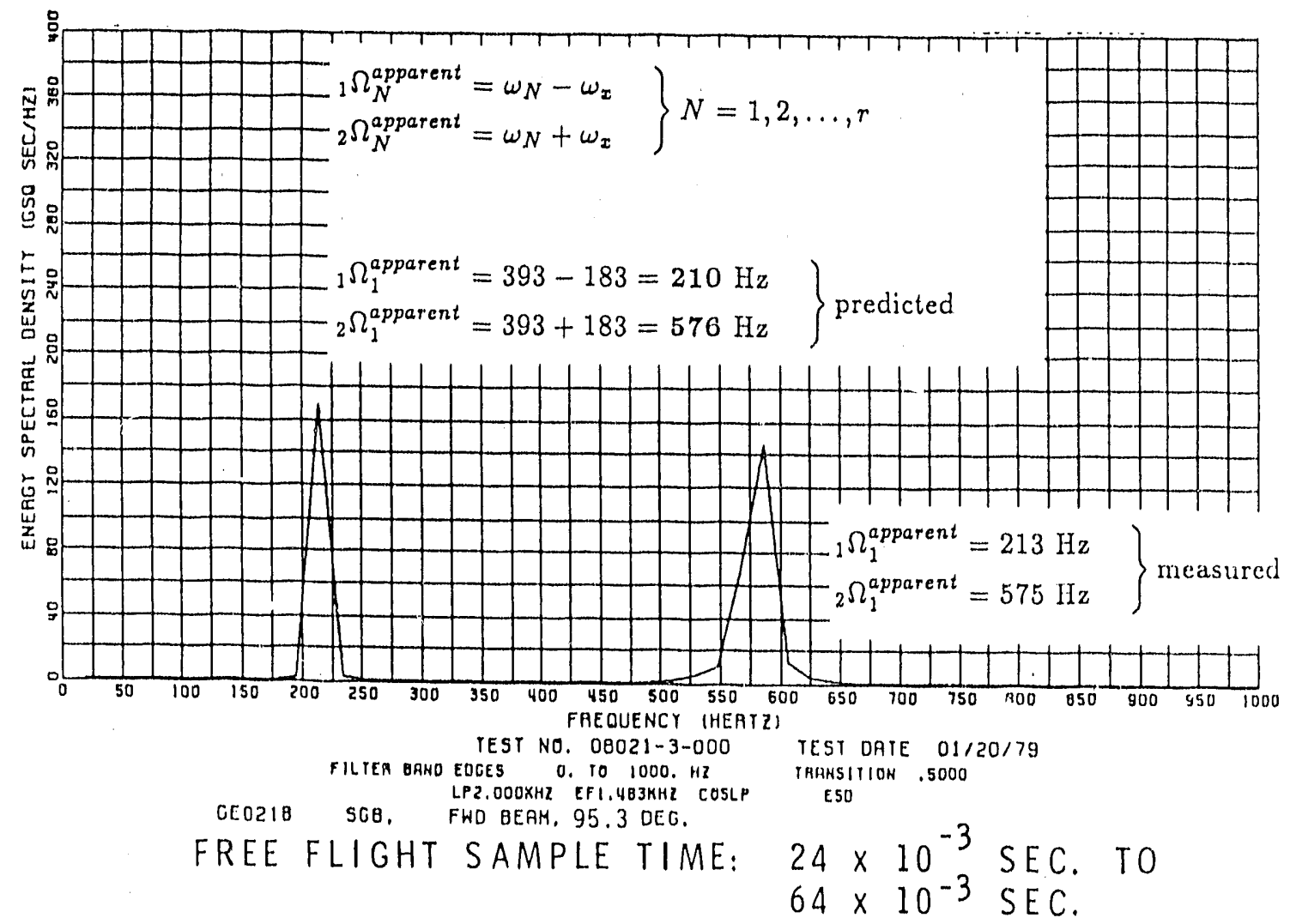

Figure 8. Measured and predicted apparent bending frequencies associated with the first mode for lateral beam vibration. As the spin speed $\omega_{x}$ gradually approaches the first bending natural frequency $\omega_{1}$, the first apparent frequency ${ }_{1} \Omega_{1}^{\text {apparent }}$ approaches zero and the second apparent frequency ${ }_{2} \Omega_{1}^{\text {apparent }}$, approaches $2 \omega_{1}$. Large-amplitude vibrations for the beam can occur when the spin speed $\omega_{x}$ approaches $\omega_{1}$ or one of the other lower-mode bending natural frequencies. The ratio of total beam mass to projectile mass is about $2.3 \times 10^{-3}$. Consequently, as $\omega_{x}$ approaches $\omega_{1}$, the flight dynamics of the projectile during free flight is not significantly affected by the beam vibration. 


\section{CONCLUSIONS}

\section{Nonrotating Beam or Cable}

When the beam is not rotating (i.e., $\omega_{x}=0$ ), dynamic buckling (divergence) occurs for the system shown in Figure 1 when the fluid speed equals or exceeds the critical fluid speed given by Eq. (29) and the inequality (30). For this case there are no oscillatory motions associated with the dynamic instability and the critical speed ratio for the fluid is independent of the damping factor. The critical speed ratio $\beta_{c r}$ (or the I indimensional fluid speed at which instability of the beam occurs) is increased by

- Decreasing $\alpha$, the nondimensional fluid mass,

- Increasing $P_{y}^{*}$, the nondimensional axial tensile force acting on the beam,

- Increasing $\gamma_{y}$, the nondimensional foundation stiffness.

Physically, $\beta_{c r}$ is increased by increasing the system stiffness and is independent of the damping factor $\xi_{N y}$.

A beam with boundary conditions such as fixed-pinned or fixed-fixed is more stiff than when simply supported. Hence, the results presented by Eq. (29) and the inequality (30) should represent a lower bound for the fluid critical speed when compared to a beam having fixed-pinned or fixed-fixed boundary conditions.

Equation (33c) gives the critical speed ratio for an incompressible, frictionless fluid flowing through a uniform fixed-fixed cable supported on a uniform elastic foundation.

If the coupled Eqs. (21) and (22) are utilized to numerically determine $\beta_{c r}$ (i.e., $V_{c r}$ ) when $\omega_{x}$ equals zero, the inequality (30) suggests that the minimum number of terms $N$ (or coupled equations) required for accurate numerical results (e.g., numerical integration of the equations of motion) is given by the first value of $N$ which satisfies the inequality $N^{2}(N+1)^{2} \geq \gamma_{y}$. Increasing $N$ above this value may not result in any appreciable decrease in the calculated value of $\beta_{c r}$. However, decreasing $N$ below this value may result in calculated values of $\beta_{c r}$ which have not converged and are significantly larger than the correct value of $\beta_{c r}$. 


\section{Rotating Beam or Cable}

For each mode $N$ of undamped lateral vibration, resonance can occur at two critical spin speeds for the beam provided the fluid speed $V$ is less than or equal to the critical fluid speed $V_{c r}$. If the fluid speed is less than the critical fluid speed, but sufficiently high, resonance can occur at very low values of spin speed. If the fluid speed exceeds the critical fluid speed, resonance occurs for any spin speed greater than zero. (Refer to Cases 1 through 4.)

During resonant vibration the two apparent frequencies for each mode of undamped lateral vibration consist of a certain combination of the two critical spin speeds associated with resonance for the $N^{\text {th }}$ mode. Refer to Eqs. (63a) and (64a) and to Cases 1 through 3 for the two critical spin speeds of interest for each mode.

The accuracy of Eqs. (44a) and (45a) and of Eqs. (63a) and (64a) can be examined by numerically integrating the coupled equations of motion [i.e., (21) and (22)] to determine the resonant lateral beam response. This can be done by specifying the parameters that appear in Eqs. (21) and (22); e.g., $\beta\left(V<V_{c r}\right), P_{y}^{*}, P_{z}^{*}, \gamma_{y}, \gamma_{z}, \alpha, \omega_{N y}, \omega_{N z}$, etc., and $\omega_{x}$. The specified value for the spin speed $\omega_{x}$ is initially chosen to correspond to a critical spin speed associated with a particular mode of lateral resonant vibration. The initial value for a critical spin speed can be estimated from Eq. (44a) or (45a). It is expected that these equations will yield good estimates of the $2 N$ beam critical spin speeds for the lower modes.

During undamped free lateral vibration, which is not associated with resonance for the spinning beam, the amplitudes of vibration in the rotating body fixed $x-y$ and $x-\cdots$ planes (refer to Figure 1) consist of an amplitude modulated sinusoidal vibration where. the amplitude is sinusoidally modulated by a combination of frequencies composed of $\omega_{x}, \Omega_{N y}$ [refer to Eqs. (40) and (34a)], and $\Omega_{N z}$ [refer to Eqs. (41) and (35a)]. Consequently, the undamped free bending vibration of the $N^{\text {th }}$ mode for the spinning beam appears to be composed of two apparent frequencies and neither of these two apparent frequencies corresponds to the $N^{\text {th }}$ natural bending frequencies, $\Omega_{N y}$ and $\Omega_{N_{z}}$, for the nonspinning beam.

\section{Discussion}

Recall that the coupled Eqs. (21) and (22) (note that the equations are coupled even when $\omega_{x}=0$ ) for the beam generalized coordinates result from using the Galerkin method to represent the motion of the beam as a $2 N$ degree-of-freedom system. Note. that these linear, second order, ordinary differential equations have constant coefficients. One of the reasons for this is that the acceleration of the fluid along the $x$ axis of the beam is zero. When this is not the case, the velocity of the fluid along the $x$ axis is a function of time and the analytical solution for the $2 N$ set of coupled equations . ith time dependent coefficients is difficult, even when the acceleration of the fluid along the $x$ axis is constant. 
When the coefficients in these equations are periodic functions of time, peculiar dynamic stability problems can arise. For example, if the acceleration of the fluid along the beam axis is zero but the density variation of the fluid is periodic with respect to time with a period $T$, the $2 N$ coupled Eqs. (21) and (22) can be expressed by ${ }^{4}$

$$
\underline{M}(t) \ddot{\bar{q}}(t)+\underline{C}(t) \dot{\bar{q}}(t)+\underline{K}(t) \bar{q}(t)=\bar{F}(t),
$$

where the mass, damping, and stiffness matrices are periodic with respect to time, each with a period $T$. That is,

$$
\begin{aligned}
& \underline{M}(t+T)=\underline{M}(t) . \\
& \underline{C}(t+T)=\underline{C}(t) \\
& \underline{K}(t+T)=\underline{K}(t) .
\end{aligned}
$$

For this case Eq. (72) is similar to a set of Hill's equations or Mathieu-Hill's equations. Consequently, multiple regions of unstable dynamic response will occur rather than a single region as indicated by Eqs. (29) or (33b). Qualitatively, these multiple regions of stable and unstable dynamic response will have the general appearance as indicated in Figure 9. With respect to Figure 9, when damping is not present, the smaller unshaded regions, associated with unstable dynamic response for the tube, will be more extensive than shown in Figure 9 and will extend to the $\beta$ axis.

For a system of equations analogous to Eqs. (72), the boundaries separating the regions of stable and unstable dynamic response can be quantitatively determined by utilizing Floquet theory [3] and an iterative computational procedure which ^ equires, for each iteration, numerically integrating the equations of motion for a time equal to the period $T$ of the coefficient matrix. However, the integration must be done $2 N$ times for the case where the spin speed $\omega_{x}$ is zero and $4 N$ times for the case where the spin speed $\omega_{x}$ is not zero [refer to Eqs. (21) and (22)]. For further information regarding Floquet theory and its application, refer to [4].

4 Another example occurs when the fluid speed is periodic. 


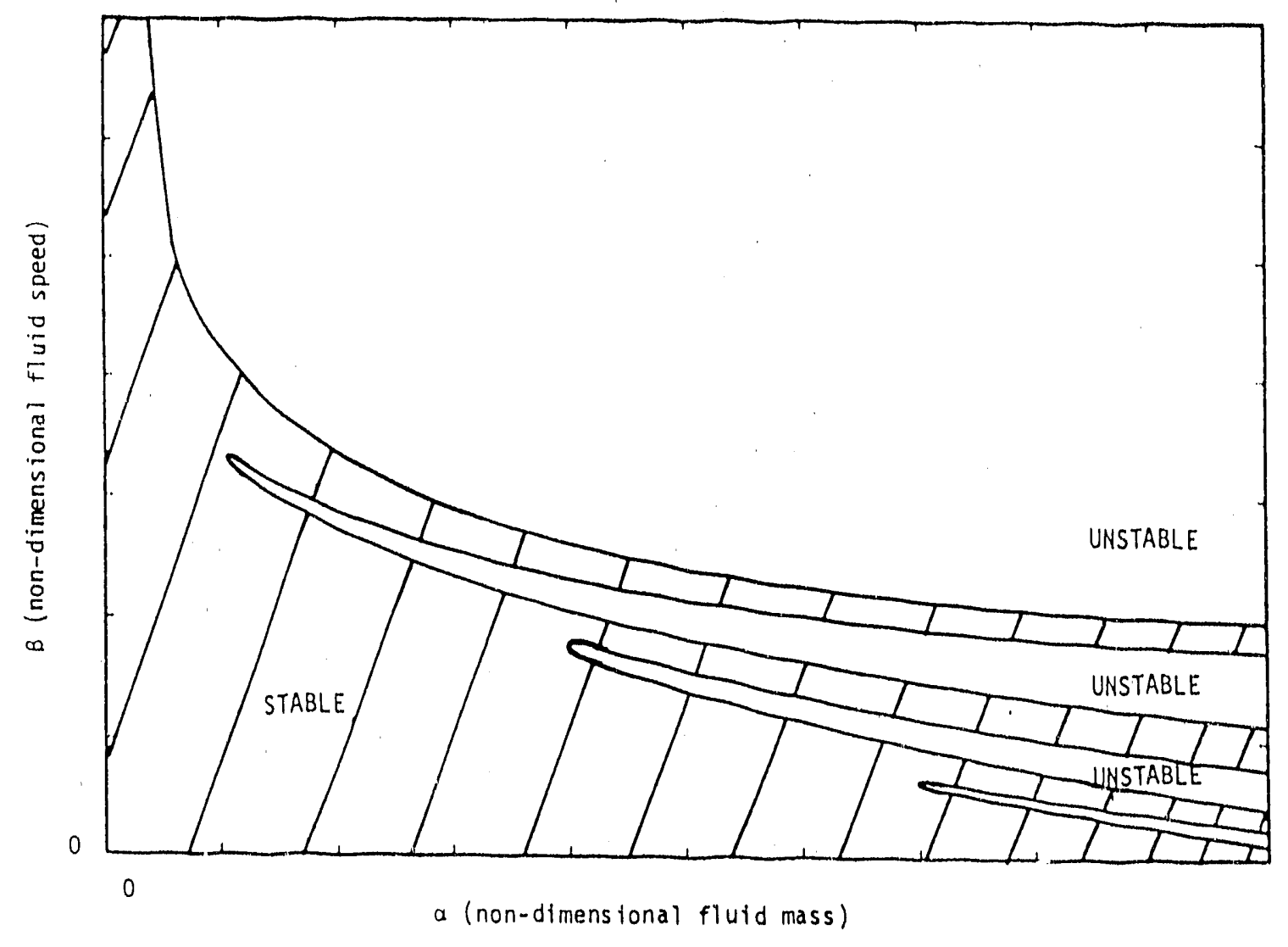

Figure 9. Qualitative representation for the regions of stable (shaded) and unstable (unshaded) dynamic response for a nonrotating, hollow tube, with viscous damping, carrying an incompressible frictionless fluid flowing at a constant speed where the density of the fluid is periodic with respect to time. 


\section{REFERENCES}

1. Benedetti, G. A., "Derivation of the Coupled Equations of Motion for a Beam Subjected to Three Translational Accelerations and Three Rotational Accelerations," SAND79-8202, February 1979.

2. Benedetti, G. A., "Dynamic Response of a Beam Subjected to Gun Launch Acceleration," SAND83-8206, April 1983.

3. Cesari, Lamberto, Asymptotic Behavior and Stability Problems in Ordinary Differential Equations, 3d ed., Springer-Verlag, New York, 1971.

4. Benedetti, G. A., "Dynamic Stability of a Spinning Tube Conveying a Flowing Fluid," SAND84-8703, February 1985. 

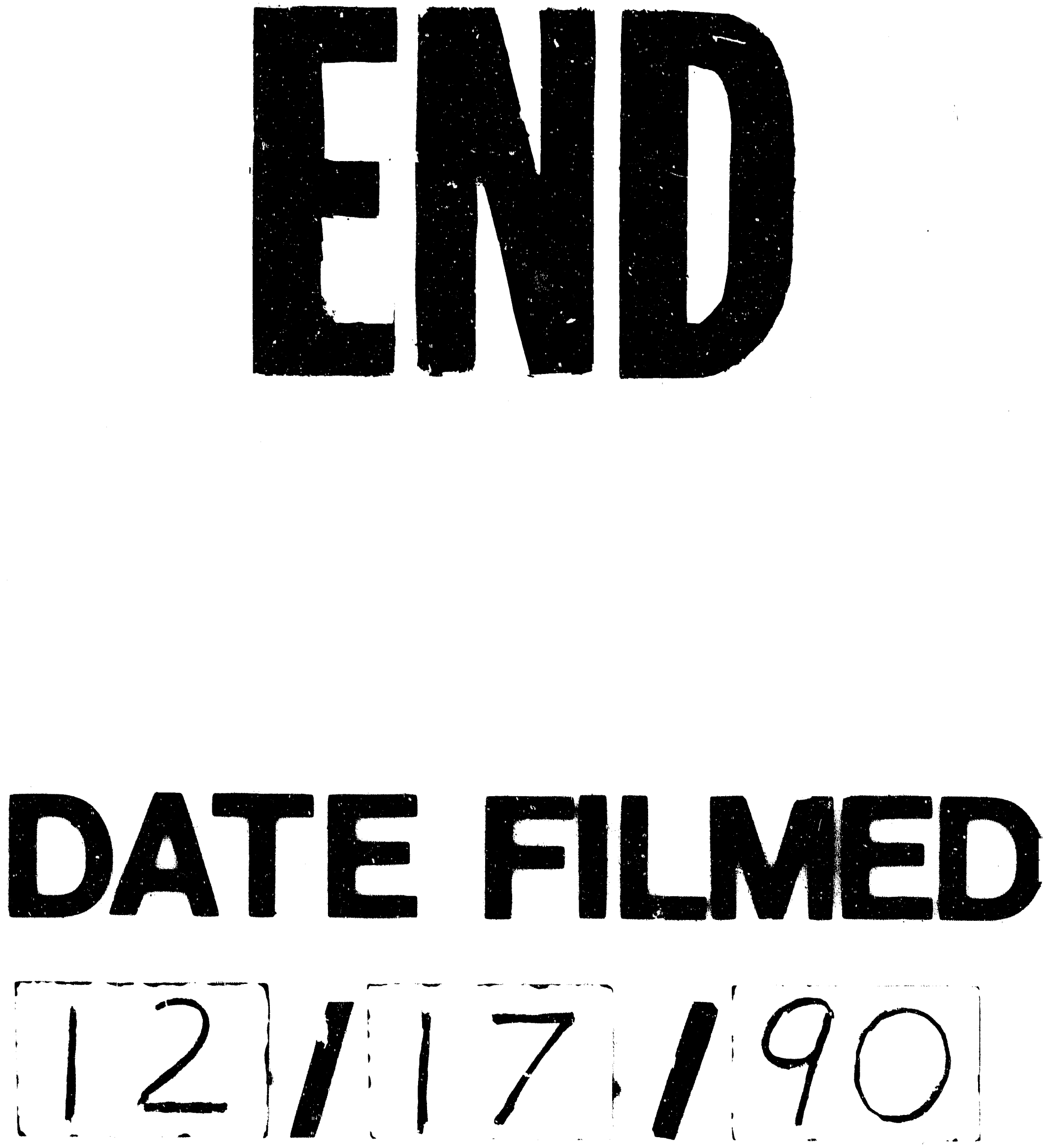
